\title{
Developments on Thermometric Techniques in Probing Micro- and Nano-heat
}

\author{
Ruijie Qian, ${ }^{1,2, \dagger}$ Xue Gong, ${ }^{1, \dagger}$ Huanyi Xue, ${ }^{1, \dagger}$ Weikang Lu, Liping Zhu ${ }^{1}$ and Zhenghua $\mathrm{An}^{1 *}$
}

Temperature is always of fundamental importance in various microscopic systems ranging from solid state nano-devices physics, chemical micro-reactions and biological cells. As traditional thermometers for macroscopic systems are not applicable in microscopic systems, alternative tools have to be developed to measure temperature at micro- and nano-scale. We provide here are view of the main currently available micro- and nano-thermometry tools including microscopic infrared thermometer, thermoreflectance, micro-Raman, plasmon energy expansion thermometry (PEET), Diamond thermometers, nanomaterial-based nanothermometers and two thermometric techniques based on scanning probe microscope (SPM), namely, scanning thermal microscope (SThM) and our recently-developed scanning noise microscope at terahertz (SNoiM).

Keywords: Temperature; Thermometry; Micro/Nano-heat

Received 30 September 2019, Accepted 16 December 2019

DOI: $10.30919 /$ esee8c369

\section{Introduction}

As one of the seven basic physical quantities, temperature is important for understanding the principles and pictures in general physical, chemical and biological processes. ${ }^{1-5}$ Although thermocouples and thermistors constitute to be accurate, affordable and easy-to-install experimental probes, these traditional thermometers for macro-scale systems are no longer applicable for micro- and nano-sized systems $(<10 \mathrm{~mm})$. Development new micro- and nano-thermometers are therefore highly demanded to explore the rich nano-science in microscopic systems in which microscopic heat generation and dissipation are generally involved. ${ }^{6-10}$ For instance, in microchips containing smaller and smaller transistors with a very high integration density, Joule heating turns out to be a bottlenecking problem in limiting further improvement of device performance and energy efficiency of post-Moore nanoelectronics; ${ }^{11-13}$ In the close vicinity or socalled thermal near-field of a hot object, energy or heat can be transferred at a rate much higher than the ideal Planck black-body radiation $^{14,15}$ and the heat conduction in microscopic size can differ from classical Fourier's law due to ballistic phonon transport; ${ }^{16-18}$ Besides, electrons in nano-devices are easily driven out of equilibrium with hosting lattice, so innovative nanothermometric techniques are needed to probe both counteracting (electron and phonon) sub-systems. ${ }^{1920}$ All

\footnotetext{
${ }^{1}$ Key Laboratory of Surface Physics, Institute of Nanoelectronic Devices and Quantum Computing, Key Laboratory of Micro and Nano Photonic Structures (Ministry of Education), Department of Physics, Fudan University, Shanghai 200433, China

${ }^{2}$ National Laboratory for Infrared Physics, Shanghai Institute of Technical Physics, The Chinese Academy of Sciences Shanghai 200083, China

*E-mail: anzhenghua@fudan.edu.cn

${ }^{t}$ These authors contribute equally to this work.
}

these phenomena related with heat in micro- and nanoscale are fascinating, and attract considerable interests from both scientific research such as fundamental understanding of thermal conductivity ${ }^{21}$ and practical applications like energy transport in batteries. ${ }^{22}$

Here we provide a review of the main currently available methods for mapping temperature profiles in micro- and nano-scale. As the thermometric technique becomes exceptionally challenging in microscopic systems, it is worth to ask what kind of physical effects can arise from a micro- or nano-hot-spot, how they can be utilized in thermometry and how sensitively they depend on temperature. As shown in Fig. 1, an immediate effect from a microscopic hot spot is infrared (IR) emission as stated by Planck's black-body law. It is therefore straightforward to develop microscopic infrared thermometer by collecting out-coming IR emission with an optical microscope. This IR microscope works in a passive mode and at much longer wavelengths than usual visible microscope. Different from this, active method can also be applied since the reflection, Raman scattering, luminescence effects upon an external excitation are all dependent on the temperature of the

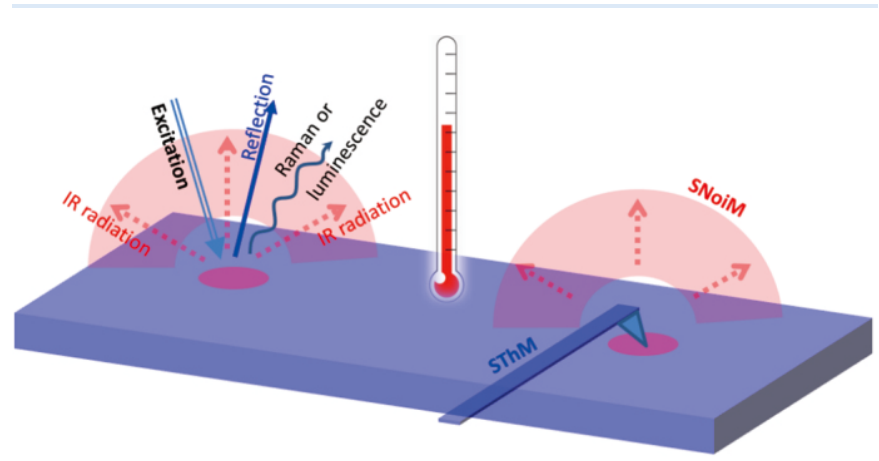

Fig. 1 Schematics of physical effects from a micro- or nano-hot-spot which can be utilized for thermometry. 
target. These effects lead to various thermometry techniques such as thermoreflectance, micro-Raman, or nanomaterial-based luminescence. In addition to the optical excitation, electron beam can also be employed and temperature-dependent plasmon can be recorded by electron energy loss spectroscopy (EELS) which results in a plasmon energy expansion thermometry (PEET). Besides, scanning probe microscope (SPM) can also be applied to realize nanothermometry: one method is to functionize the tip with thermocouple or thermistor so that the tip can work as a nano-thermometer and can be scanned directly on the sample surfaces. This technique is so called scanning thermal microscope (SThM); another approach is to use the tip to scatter in the thermal near-field the super-Planckian radiation from the hot spot and we term this technique as scanning noise microscope (SNoiM) since the near-field radiation arises essentially from the fluctuating electromagnetic (EM) fields or alternatively EM noise (either thermal
Johnson noise for thermal equilibrium system or shot noise for nonequilibrium system). We describe the working principles of the above thermometry techniques and discuss their pros and cons together with typical application examples.

\section{Microscopic infrared thermometer}

\subsection{Principle of microscopic infrared thermometer}

Any objects with finite temperatures can radiate electromagnetic waves and the intensity is determined by their temperature and emissivity. The infrared thermometer collects the electromagnetic waves at infrared wavelength and the temperature of sample can be extracted according to Planck's blackbody radiation law. To map the temperature distribution of a sample surface, infrared array detectors are used in the infrared microscope (Fig. 2(a)), and each pixel converts infrared radiation into a change in the resistance of individual pixel, which then can be recorded (a)

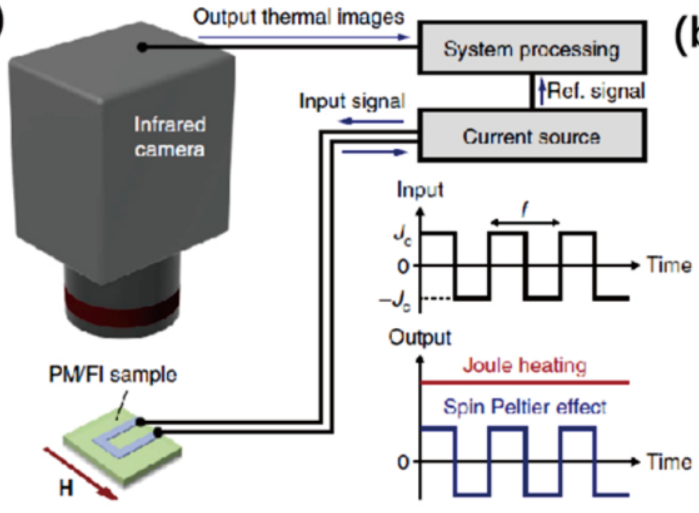

(b)

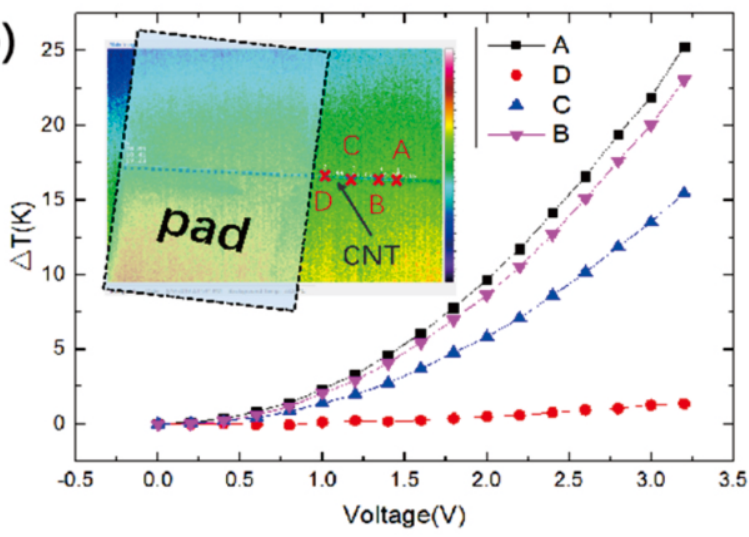

(c)
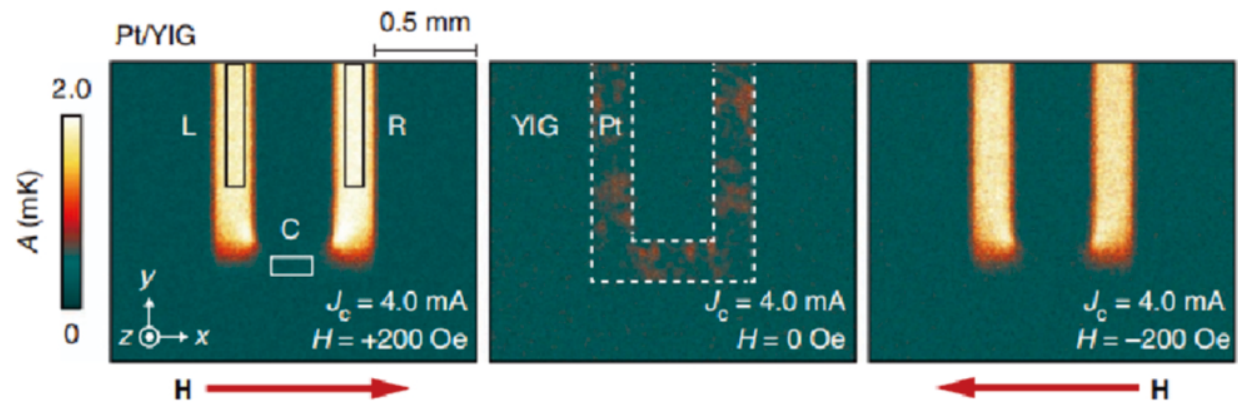

(d)
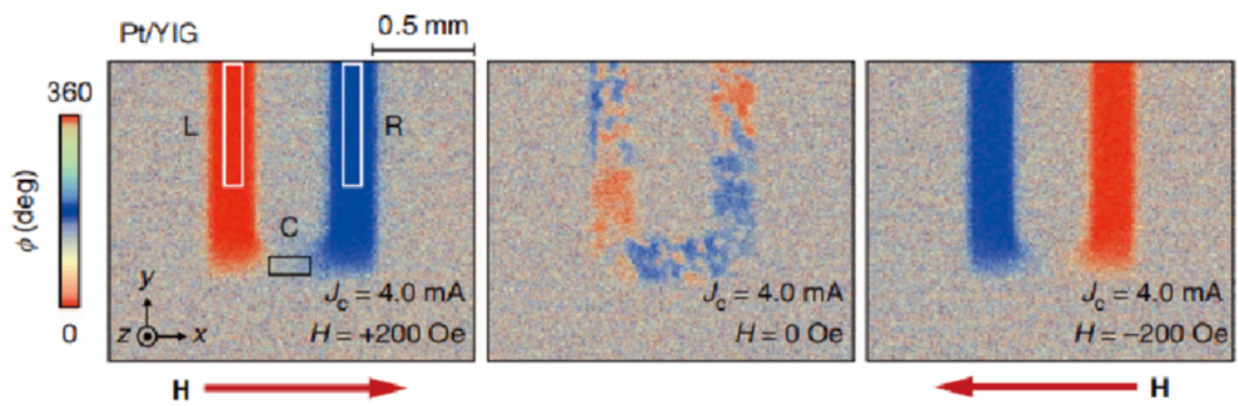

Fig. 2 (a) Schematic of the microscopic infrared thermometer setup with lock-in technique for imaging of temperature distribution due to Joule heat and Peltier effect, ${ }^{25}$ (b) temperature increase of a CNT fiber as a function of external bias at different positions (A, B, C and D), the inset depicts the twodimensional temperature mapping; (c) amplitude and (d) phase images of results at different magnetic fields $\left(\mathrm{H}=0\right.$, \pm 200 Oe). ${ }^{25}$ Reprinted with permission from Ref. [25]. Copyright 2016, Springer Nature. 
by computer and transformed into two-dimensional temperature distributions. Being similar to the optical microscope in visible region, this technique is a non-contact, non-destructive and convenient method with extraordinarily fast imaging capability. On the other hand, however, the spatial resolution of this optical method is inherently restricted by the optical diffraction limit, which is typically a few microns since the infrared wavelength is rather long compared to visible light. The temperature resolution is mainly determined by the detector sensitivity and hence cooled photon detectors are typically preferred when a high temperature sensitivity is aimed. The temperature resolution is described by Noise Equivalent Temperature Difference (NETD), which specifies the smallest temperature difference that can be detected. NETD can now reach the order of $\sim \mathrm{mK}$ and is also affected by various other factors, such as the materials and structures of infrared detectors, the detection wavelength and so on. It is worthy to mention that the absolute temperature determination depends on the accuracy of the emissivity value which is often uncertain and therefore often requires new detection mechanisms such as dual-band infrared imaging.

\subsection{Applications of microscopic infrared thermometer}

As a fast and convenient way for temperature analysis in micro-scale, infrared thermometer and imaging has been widely applied in many areas, such as in physics, electrical engineer, medicine and biology, etc. ${ }^{23,24}$ Regardless of materials and experimental conditions, temperature distribution generated by either thermal agitation, optical excitation or electrical stimulation can be mapped on different sample surfaces of no matter metals, semiconductors, insulator or biological specimen. Also thermal resistance or conductance can be possibly evaluated from the results obtained with microscopic infrared thermometer. We use a commercial microscopic infrared thermometer which has a spatial resolution of about $\sim 5$ microns and a temperature sensitivity of about 40 $\mathrm{mK}$. The temperature sensitivity can be improved to less than $1 \mathrm{mK}$ with lock-in technique, ${ }^{25}$ as schematically shown in Fig. 2(a). Fig. 2(b) displays the imaging result of a carbon nanotube (CNT) fiber with a diameter of $\sim 500 \mathrm{~nm}$ under external bias which provides the twodimensional temperature distribution due to Joule heating. The CNT fiber is heated after a bias applied, and there is a quadric relationship between temperature increase and external bias. The non-uniform temperature profile along the $\mathrm{CNT}$ arises from the energy balance between heat generation and heat dissipation at each point (A, B, C and D), from which the thermal conduction property of CNT fiber can be extracted.

The outstanding temperature sensitivity of lock-in infrared thermometer facilitates the experimental observation of many precise and fascinating thermal signals such as thermoelectric spin-Peltier effect (SPE) from which temperature difference can be induced by spin currents. ${ }^{25}$ As shown in Fig. 2(c) and (d), in a U shape device with the paramagnetic metal $\mathrm{Pt}$ fabricated on ferromagnetic insulator yttrium iron garnet (YIG), the spin current direction in vertical axis differs from each other depending on the direction of moving electrons under a global magnetic field. The spin current with different vertical directions causes the difference temperature change on the left and right arms of the U-shape sample. The high temperature sensitivity is essential to extract the tiny temperature difference $(\sim 2 \mathrm{mK})$ from the large background of Joule heat, which is realized by using sensitive microbolometers and lock-in thermography. Some other interesting phenomenon in spintronics like unidirectional spin heat conveyer effect $^{26}$ and anisotropic magneto-Peltier effect ${ }^{27}$ have also been successfully observed with infrared thermometer. As a result, infrared thermometer turns out to be an important tool for further development of this research field. On the other hand, however, study of spin behavior at smaller nano-scale is impeded by the poor spatial resolution $(\sim 10 \mu \mathrm{m})$ of far-field infrared thermometer. To overcome the diffraction-limit induced poor spatial resolution, it is advantageous to consider optical microscope with much shorter wavelength as discussed in the following sections, although an active mode has to be applied.

\section{Thermoreflectance thermometry}

\subsection{Principle of thermoreflectance thermometry}

Under an external illumination, it is natural to check the reflection first to know its temperature dependence. Although the temperature dependence of reflection does exist, it appears rather weak and therefore requires additional technique such as modulation spectroscopy to achieve reasonable performance. Modulation spectroscopy started in $1960 \mathrm{~s}^{28}$ and has been proved to be of great contribution to observe various samples including different micro- and nanostructures in research and some actual structures in industries like high electron mobility transistor (HEMT) and microelectromechanical systems (MEMS). Thermoreflectance thermometry, one of the modulation spectroscopy techniques, detects temperature variations of sample surface by measuring the optical reflectance correlated with temperature. Typically the sample temperature is modulated through controllable experimental condition and the changes in reflectivity is recorded with lock-in technique, as shown in Fig. 3(a).

The modulation can be done either externally through electromodulation (an electric field), thermomodulation (temperature) or piezomodulation (stress), ${ }^{29.33}$ or internally by periodically changing the wavelength or polarization condition of incident light. ${ }^{34}$ Although modulation spectroscopy measures the change of the complex dielectric function whose real and imaginary parts are correlated through Kramer-Kronig relations, the induced reflectivity change $\Delta R$ can be approximately written as,

$$
\frac{\Delta R}{R}=\frac{1}{R} \frac{\partial R}{\partial T}=\Delta T=k \Delta T
$$

where $k$ is the thermoreflectance calibration coefficient, typically on the order of $10^{-2}-10^{-5} \mathrm{~K}^{-1}$. Due to its differential nature, modulation reflectance experiment has the potential to suppress broad background features and therefore a high sensitivity can be reached. During the measurements of electronic devices, reflectivity at a specific range of wavelength is recorded. The relative change in reflectance generated by external alternating current may modulate at an extremely small amplitude with the order around $\sim 10^{-4} .35-37$ While the investigation is achieved with the laser, so the spot size of the excitation laser directly determine the spatial resolution. The smallest spot size for $630 \mathrm{~nm}$ probe beam wavelength is diffraction limited giving spot diameter of about $0.5 \mu \mathrm{m}$ which is better than infrared thermometry in previous section. Temperature resolution of thermoreflectance thermometry is typically better than $1 \mathrm{~K}^{38}$

\subsection{Applications of thermoreflectance thermometry}

Thermoreflectance can be applied widely in solid state electronics to provide information on heat dissipation and failure analysis in these complex devices. Fig. 3(b) and (c) display the standard and CCD-based thermoreflectance of a quantum cascade laser at the same $6 \mathrm{kA} \mathrm{cm}^{-2} / 20$ $\mu \mathrm{s} / 20 \mathrm{kHz}$ working condition. ${ }^{39}$ The CCD thermoreflectance has many potential applications, being especially well suited for small size devices, Fig. 3(d) and (e) show the thermoreflectance images (d) and temperature maps (e) of a $\mathrm{MoS}_{2}$ field-effect-transistors (FETs) with large drain current at power densities from $0.6 \mathrm{~W} \mathrm{~mm}^{-1}$ to $1.8 \mathrm{~W} \mathrm{~mm}^{-1}$. 
These thermometric imaging results suggest that the self-heating effect may play an important role in microelectronics and optoelectronics and has to be taken into account.

\section{Micro-Raman thermometry}

\subsection{Principle of Micro-Raman thermometry}

When a material is illuminated, the excitation photons can be elastically (Rayleigh) or inelastically (Raman) scattered. Micro-Raman thermometry (Fig. 4(a) and (b)) examines the inelastic scattering processes in a crystal, in which the incident photons are scattered by phonons leading to a shift in energies of the out scattered photons with creating or annihilating an optical phonon. The probability of the inelastic scattering is temperature-dependent and is related to the occupation number of optical phonons. ${ }^{36,42}$ Measurement of the crystal lattice temperature is hence given often by the intensity ratio of the Stokes (S) and anti-Stokes (AS) lines or sometimes by their spectral shift relative to the incident excitation light. ${ }^{43-46}$ The measured temperatures can be determined by analyzing the S-to-AS intensity ratio of the corresponding phonon peak (e.g., transverse-optical (TO) in GaAs) employing the expression $\mathrm{I}_{S} \mathrm{I}_{\mathrm{AS}} \approx \mathrm{C} \exp (\hbar \Omega / \mathrm{kT})$, where $\mathrm{I}_{S}, \mathrm{I}_{A S}$ are the $\mathrm{S}$ and AS phonon peak intensities, $\hbar \Omega$ is the phonon energy $(\sim 261$ $\mathrm{cm}^{-1}$ at room temperature for $\mathrm{TO}$ phonon in GaAs), and $\mathrm{T}$ is the lattice
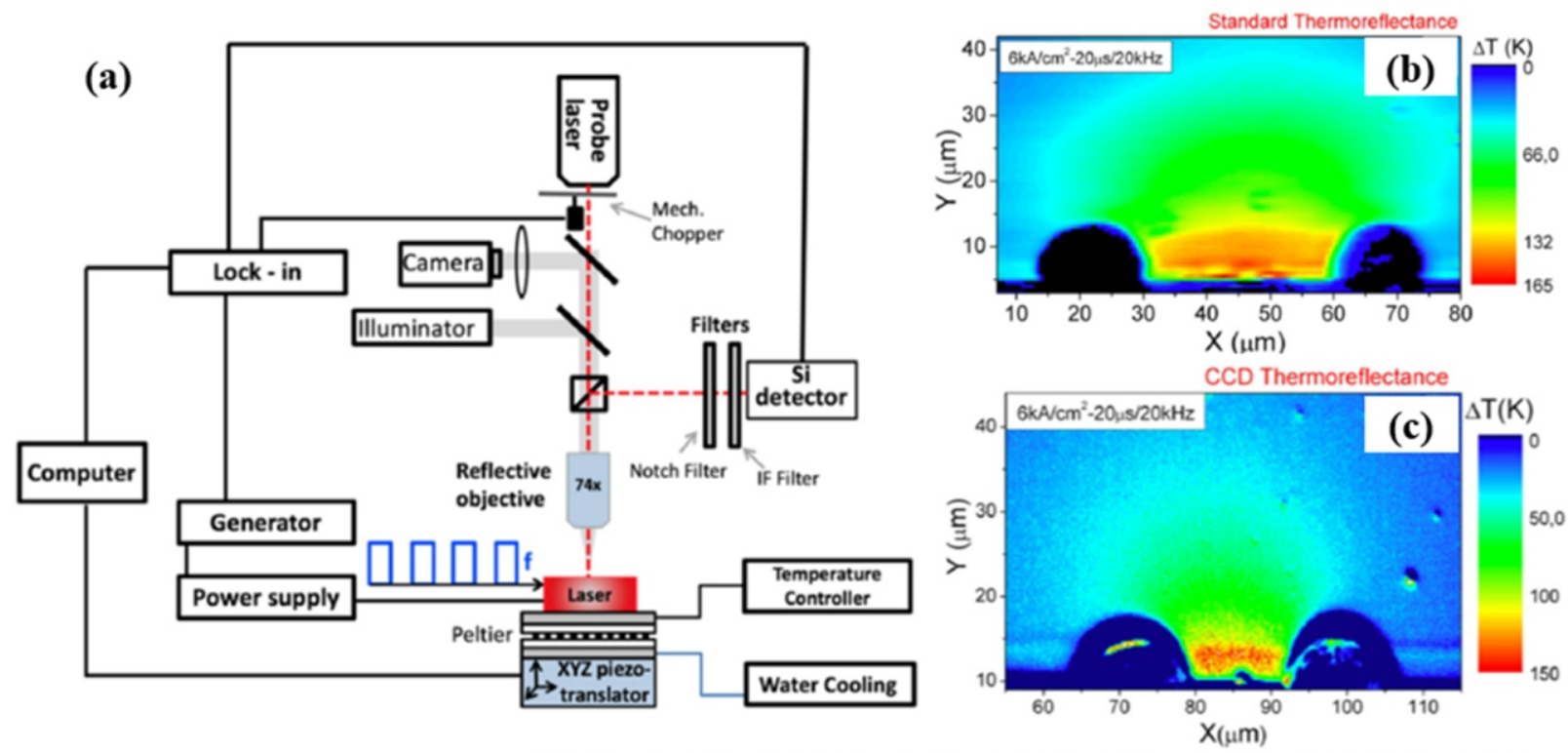

(d)
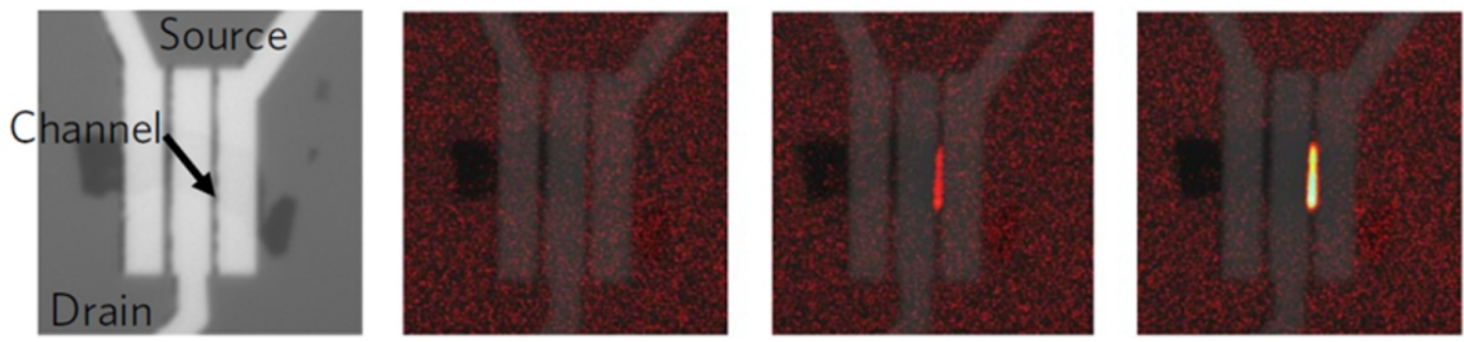

(e)
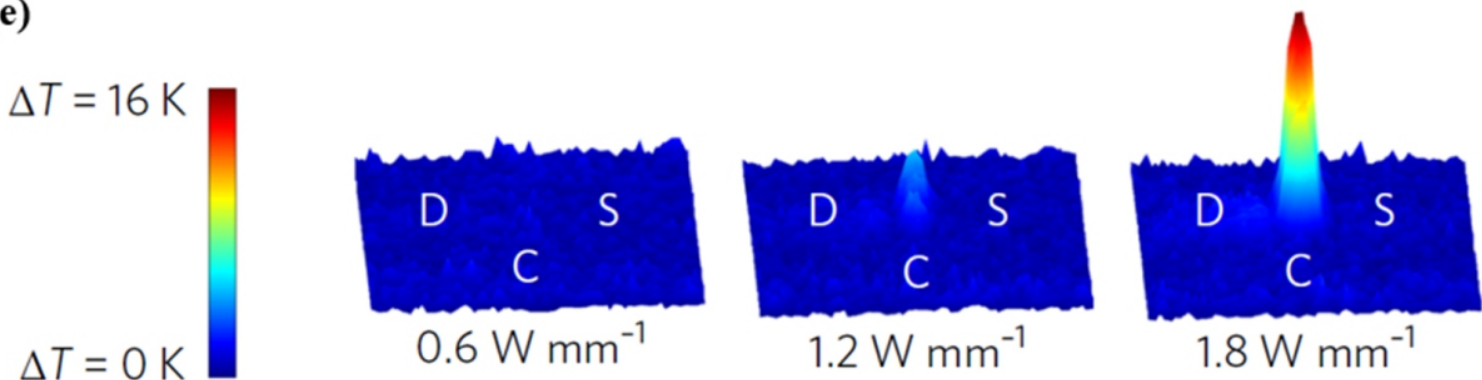

Fig. 3 (a) Experimental set-up for thermoreflectance mapping of laser and laser bar mirrors. Reproduced from Ref.[39]. (b) and (c) Temperature distribution maps registered by standard thermoreflectance spectroscopy (b) and CCD-thermoreflectance (c) of a quantum cascade laser at the same $6 \mathrm{kA}$ $\mathrm{cm}^{-2} / 20 \mu \mathrm{s} / 20 \mathrm{kHz}$ working condition. 2016 IOP Publishing. Reproduced with permission from Ref. [39]. All rights reserved. Thermoreflectance images (d) and temperature maps (e) of a MoS 2 FETs with large drain current at power densities from $0.6 \mathrm{~W} \mathrm{~mm}^{-1}$ to $1.8 \mathrm{~W}^{-1} \mathrm{~mm}^{-40}$ Reprinted with permission from Ref. [40]. Copyright 2017, Springer Nature. 
temperature, $\mathrm{C}$ is a correction factor for the frequency-dependent response of the detection system. The value of $\mathrm{C}$ is derived from the $\mathrm{S}-$ to-AS intensity ratio of the phonon from a reference substrate with same material at a fixed temperature (e.g., GaAs at room temperature). With micro-Raman method, temperature resolution of $\sim 5-10 \mathrm{~K}$ are given with spatial resolution depending on the excitation laser spot size and can be below $1 \mu \mathrm{m},{ }^{36}$ which is similar to thermoreflectance thermometry (section 3) but is improved considerably compared to the previous passive infrared thermometry with much longer wavelength (section 2).

\subsection{Applications of Micro-Raman thermometry}

Micro-Raman thermometry technique is suitable to measure the inplane temperature distribution and related thermal properties of bulk materials, thin films and nanowires. It has been used for measuring various thermoelectric materials with different dimensions, such as silicon/germanium wafers, ${ }^{47}$ alumina thin films, ${ }^{48}$ suspended graphene ${ }^{49,50}$ bulk nanocrystalline silicon, GaAs nanowires, ${ }^{51}$ and $\mathrm{Si}$ nanowires, ${ }^{52}$ among others. Furthermore, some other photovoltaic measurements can be observed with this technique. ${ }^{52}$ When the thickness of the film is on the order of its optical absorption coefficient such that the heating can be considered independent of the depth in the film, micro-Raman thermometry allows direct determination of thermal conductivity from steady-state measurement despite of a moderated accuracy of $\sim 20 \%$.

Fig. 4(c) shows a Raman temperature map of a suspended film across a trench in y direction. In the center suspended part, the Raman temperature reaches as high as $\sim 700 \mathrm{~K}$, whereas the direct contact part with the supporting Ge substrate can conduct the heat efficiently to the underlying heat sink, so that the Raman temperature stays close to room temperature. Fig. 4(d) shows the same data in a more quantitative way.
For different scans across the trench, the central Raman temperature varies by approximately $10 \%$, which gives the same estimate for the accuracy of thermal conductivity.

The resolution of the above three optical thermometry (infrared, thermoreflectance and micro-Raman) is eventually limited by the diffraction and great efforts have to be spent to overcome this limit and reach super-resolution in thermometry imaging.

\section{Plasmon energy expansion thermometry (PEET)}

One solution to improve the spatial resolution is to utilize the electron microscope since the wavelength of high-energy electron beam is much shorter. It is however nontrivial to find a proper mechanism with applicable temperature dependence in electron microscope. Mecklenburg et al. ${ }^{54}$ reported an electron-microscope-based thermometry through a mechanism analogous to the optical micro-Raman technique, namely, inelastic scattering of the incident light or electron beam. This new noncontact method is called plasmon energy expansion thermometry (PEET) and can measure bulk temperature with nanoscale spatial resolution. As schematically shown in Fig. 5(a), it combines scanning transmission electron microscope (STEM) and electron energy loss spectroscopy (EELS), while it has a negligible impact on the measured sample temperature. When an electron beam created by STEM penetrates a sample, a bulk plasmon with energy $E$ will be excited. According to the free-electron model, this energy is proportional to the square root of the valence electron density $(n)$ of sample and can be correspondingly detected by EELS. ${ }^{5457}$ As thermal expansion changes this electron density and thereby changes the plasmon energy, the temperature can be deduced from the plasmon energy shift measured by EELS and vice versa.

Two typical EELS spectra taken at two different temperatures are
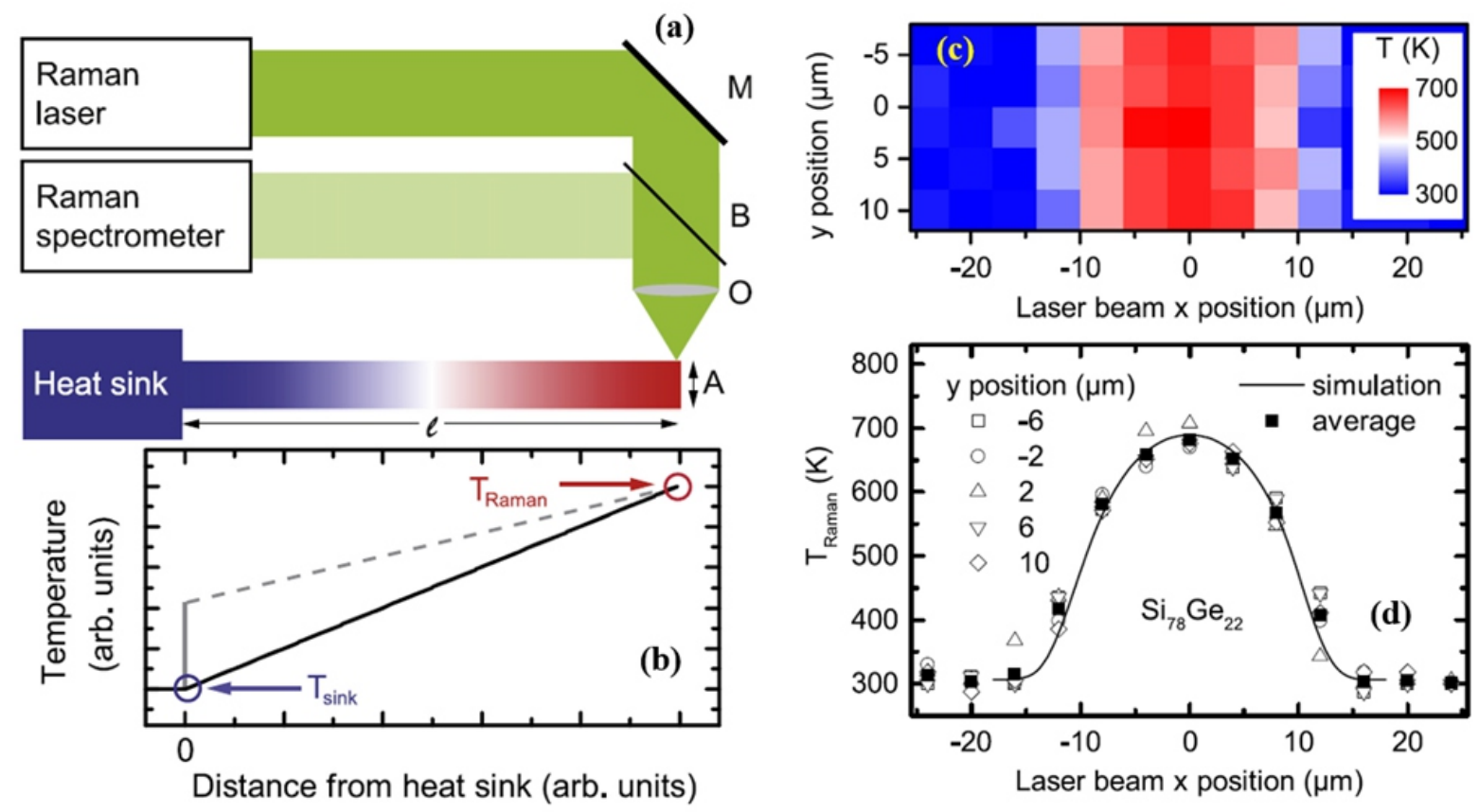

Fig. 4 (a) Micro-Raman thermometry for measuring the thermal conductivity of a bar-shaped material. The Raman laser acts both as the heating source and, together with the Raman spectrometer, as the thermometer. The beam of the laser is directed by mirrors (M) to the microscope objective (O), which focuses the light on the sample of length $l$ and cross section A. Raman scattered light is directed via abeam splitter (B) to the Raman spectrometer and Raman signal is measured. (c) Color coded Raman temperature map of the film on the trench. (d) Raman temperature scans across the trench, together with the simulation of the Raman temperatures, shown as a solid line. ${ }^{47}$ (C)2014 IOP Publishing. Reproduced with permission from Ref[47]. All rights reserved. 
shown in Fig. 5(b). As illustrated, when the local temperature increased from $293 \mathrm{~K}$ (black) to $413 \mathrm{~K}$ (red), the corresponding small plasmon peak shift, roughly $-0.54 \mathrm{meV} / \mathrm{K}$, is measured by the curve fitting of the peak maximum. The plasmon energy can be regarded as the energy difference between the zero loss peak and the plasmon peak. By scanning STEM electron beam over the sample and measuring the plasmon energy at each position, the plasmon energy map can be acquired. Fig. 5(c) and 5(d) show the plasmon energy maps of an

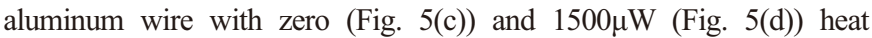
power applied, respectively. In both two energy maps, the sensitive curve-fitting procedure ensure the nanoscale resolution, in which the grain boundaries and the surface details are clearly visible (as shown in Fig. 5(c) and 5(d)). Combining the two plasmon energy maps, the temperature distribution can be deduced from the peak shift at each pixel. The spectra acquisition rate was about $76 \mathrm{~Hz}$ for the data in Fig. 5(d). The standard deviation of the measured temperature in various small regions ranges from 20 to $30 \mathrm{~K}$, which is equivalent to a temperature sensitivity of about $3 \mathrm{~K} / \mathrm{Hz}^{54},{ }^{58}$ A variety of spectra acquisition rates yielded this same figure of merit. From this work, it is interesting to note that this PEET thermometer works more or less like Fahrenheit's mercury-in-glass thermometer and achieves its sensitivity and accuracy based on the calibrated thermal expansion.

In principle, the spatial resolution of PEET is dominated by the electron beam which has a typical dimension of $1 \sim 2 \mathrm{~nm}$, therefore the spatial resolution can be much better than previous optical thermometry techniques. On the other hand, however, it is necessary to have vacuum condition and sophisticated electron microscope with EELS setup, and hence inconvenient to be applied in wider areas. In addition to electronmicroscope-based thermometry, alternative approaches to reach better spatial resolution are to utilize nano-sized particles or tips as localized thermometric probes, as we discuss in next sections.

\section{Diamond thermometry}

6.1 The NV center and diamond thermometry

Diamond with imbedded nitrogen-vacancy (NV) centers has attracted considerable attention in many fields due to its exotic properties, including magnetic/electric-field sensing, ${ }^{59,60}$ angular-rate measurement, ${ }^{61}$ and bio-imaging applications. ${ }^{62}$ The $\mathrm{NV}$ center is a negatively charged point defect in diamond, consisting of a nitrogen atom and a vacancy that replaces the two adjacent carbon atoms (Fig. 6(a)). ${ }^{63}$ The NV center can be regarded as a two-energy-level system (Fig. 6(b)) and function as an optically addressable spin qubit with long coherence time. ${ }^{6466}$ Since the properties of NV center are very sensitive to the local lattice thermal expansion due to temperature variation, ${ }^{67-69}$ it can work as a highly sensitive thermometer. Acosta et $a .^{70}$ were the first to investigate the temperature dependence of magnetic-resonance spectra of $\mathrm{NV}^{-}$center in the range of $280 \mathrm{~K}$ to $330 \mathrm{~K}$ by an optical method. The transition frequency between two energy levels is typically in
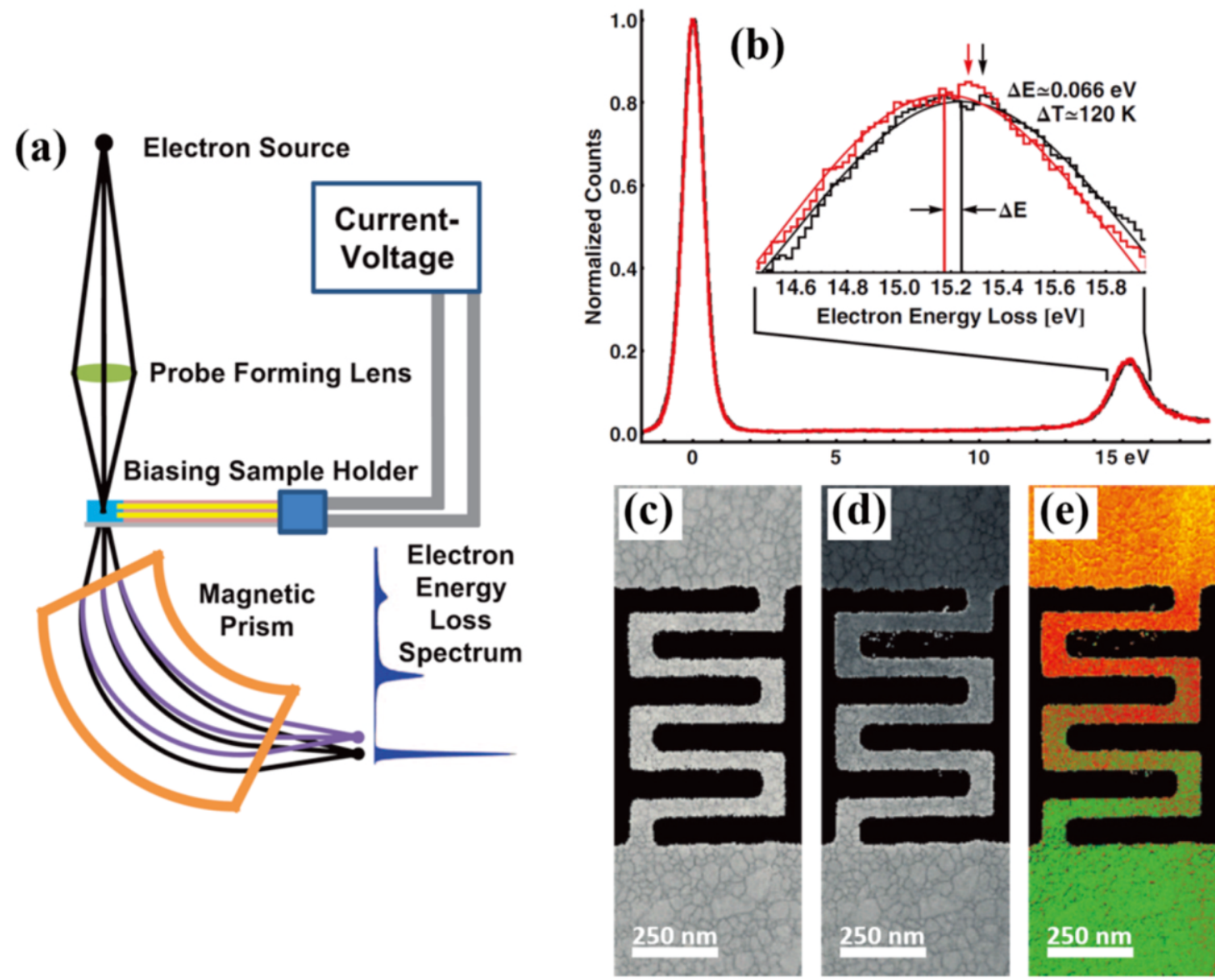

Fig. 5 (a) Schematic of the PEET instrument that based on STEM and EELS. (b) Two typical EELS spectra taken at $293 \mathrm{~K}$ (black) and $413 \mathrm{~K}$ (red), respectively. The fitted plasmon peak maxima are indicated by two arrows. (c) and (d) Plasmon energy maps of an aluminum wire with zero (c) and nonzero (d) power applied to the heater, respectively. (e) Temperature mapping deduced from (c) and (d). ${ }^{54}$ From [54], Reprinted with permission from AAAS. 
microwave range and the accurate measurement of this temperaturedependent transition frequency is prerequisite for precise temperature measurement. For all diamond samples with different $\mathrm{NV}^{-}$center concentrations (from $10 \mathrm{ppb} \sim 15 \mathrm{ppm}$ ), the zero-field splitting $D$ was found to vary sensitively with a temperature coefficient of $d D / d T=-74.2(7) \mathrm{kHz} / \mathrm{K}$. The large $d D / d T$ coefficient and the sharp magnetic resonance peak of the $\mathrm{NV}^{-}$center make diamond with $\mathrm{NV}^{-}$ center a promising candidate for the high-precision thermometry at around room temperature. Later, Chen et al. ${ }^{71}$ and Toyli et al. ${ }^{72}$ investigated the temperature-dependence magnetic resonance and fluorescence spectra of $\mathrm{NV}$ center in two broad temperature ranges of $5.6 \mathrm{~K}$ to $295 \mathrm{~K}$ and $300 \mathrm{~K}$ to $700 \mathrm{~K}$, respectively. Remarkably, the thermal sensitivity between room temperature and $600 \mathrm{~K}$ quantified by Toyli et al. was about $100 \mathrm{mK} / / \mathrm{Hz}$, and may be further increased to one order of magnitudes for $\mathrm{NV}$ center imbedded in the pure diamond. ${ }^{73-75}$ Kucsko et $a l^{74}$ used a bulk diamond sample with isotopically pure carbon-12 isotope to suppress the magnetic perturbation from carbon-13 nuclear spin. Utilizing such materials and a modified spin-echo sequence method, they detected temperature variations with sensitivity up to $1.8 \mathrm{mK}$ under ideal experimental conditions. Other groups have also achieved high thermal sensitivity at this similar level using analogous experimental methods and conditions. Temperature sensitivity of bulk diamond thermometry can be further improved to a thermal sensitivity of 10.1 $\mathrm{mK} / \mathrm{Hz}$ by Thermal Ramsey (T-Ramsey) method. ${ }^{75}$

\subsection{Nanodiamond thermometer}

As illustrated above, diamond bulk materials with NV centers can be applied as highly sensitive thermometers in a wide temperature range. However, in order to achieve a high spatial resolution of temperature detection by NV center at the nanoscale, bulk diamonds are not suitable. Plakhotnik and Gruber $^{76}$ proved the feasibility of diamond nanothermometers for the first time and experimentally investigated the temperature-dependent photoluminescence of NV center in nanodiamonds with a size of 20 35 $\mathrm{nm}$. It was found that when the temperature changed from $300 \mathrm{~K}$ to $670 \mathrm{~K}$, the luminescence intensity of $\mathrm{NV}$ centers in nanodiamonds decreased to a quarter. This work supports the feasible application of diamond nanothermometers with high spatial $(\sim 10 \mathrm{~nm})$ and temporal $(\sim 100 \mathrm{~ms})$ resolutions in a wide temperature range.

The first practical application of nanodiamond thermometers was reported by Kucsko et al. in 2013. ${ }^{74}$ They used an ingenious nanowireassisted delivery technique to induce nanodiamonds and gold nanoparticles (both $100 \mathrm{~nm}$ ) into cells (Fig. 6(c) and (d)). When gold nanoparticles are activated by laser beams, they become the local heat sources within the cell(Fig. 6(c)). The combination of nanodiamond (a)
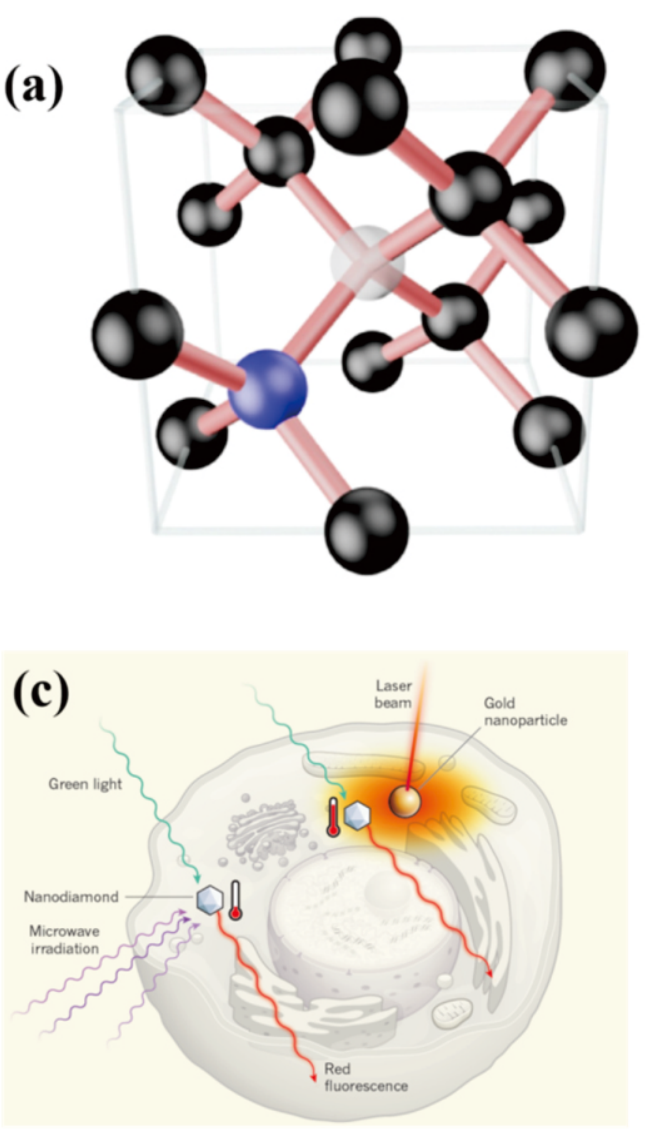

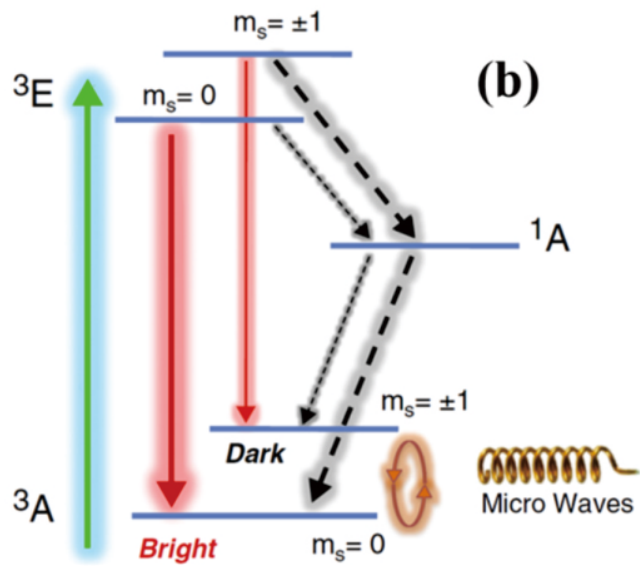

( $\times 10^{4}$ c.p.s.)

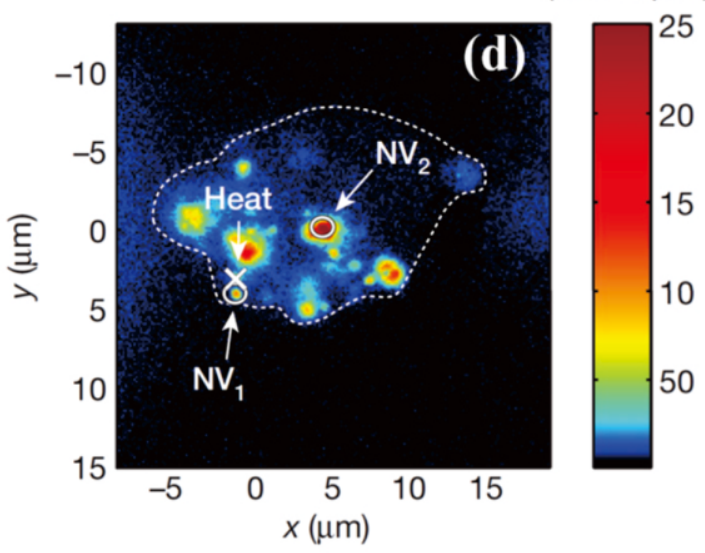

Fig. 6 Properties of NV center. (a) The atomic structure of a single NV center in diamond lattice. The blue, white, and black spheres represent the nitrogen, vacancy, and carbon atoms, respectively. (b) Energy level diagram with different spin states of NV center. ${ }^{62}$ Reprinted from Ref[62], Copyright 2014, With permission from Elsevier. (c) Schematic of NV centers within nanodiamond for monitoring temperature in living cells. ${ }^{125}$ Reprinted with permission from Ref. [125]. Copyright 2013, Springer Nature. (d) Confocal scan of a single cell under laser lamination. $\mathrm{NV}_{1}$ and $\mathrm{NV}_{2}$ represent two nanodiamond positions with different distance from the gold particle (marked by cross). ${ }^{74}$ Reprinted by permission from Ref. [74]. Copyright 2015, American Chemical Society. 
thermometers and gold nanoparticles allows to both monitor and control the temperature at the nanoscale. These two types of nanoparticles can be imaged by a confocal microscope with a resolution of about $100 \mathrm{~nm}$. Two $\mathrm{NV}$ positions (marked by $\mathrm{NV}_{1}$ and $\mathrm{NV}_{2}$ ) at different distances from the gold nanoheater were chosen to investigate the temperature variations with the incident laser power. As illustrated in Fig. 6(d), the $\mathrm{NV}_{1}$ position, which is closer to the heater than $\mathrm{NV}_{2}$, shows a stronger temperature dependence of the laser power. By increasing the laser power to $12 \mu \mathrm{W}$, the temperature differences of up to $3 \mathrm{~K}$ between $\mathrm{NV}_{1}$ and $\mathrm{NV}_{2}$ can be induced. This work demonstrated temperature measurement inside a single cell with sub-Kelvin sensitivity $(\sim 80 \mu \mathrm{K} / \mathrm{Hz})$ and high spatial resolution (100 $\mathrm{nm}$ level).

\subsection{Scanning Nanodiamond Microscopy}

In order to obtain the spatial distribution of temperature profiles over large area, the thermometry method proposed by Kucsko et al. will not be applicable, due to the random and discrete distribution of nanodiamond thermometers. Tetienne et $a l^{76}$ attached a nanodiamond thermometer $(\sim 100 \mathrm{~nm})$ to the tip of an atomic force microscope (AFM), which enabled the probe to scan relative to the sample while precisely controlling the distance between the sample and the tip (Fig. $7(\mathrm{a}))^{77-79}$ This approach provides up to an order of magnitude gain in acquisition time while preserving a sub-100 $\mathrm{nm}$ spatial resolution both for the topography and temperature. Fig. 7(b) shows the fluorescence image of the gold nanoheater $(\sim 40 \mathrm{~nm})$ as indicated by the bright spot. To acquire a temperature map, a full spin resonance spectrum is recorded at each pixel (as illustrated in Fig. 7(c)) of the scan at a constant incident laser power, from which the splitting parameter $D$ can be deduced for each pixel and hence the resulting temperature map can be obtained (Fig. 7(d)).

\section{Nanomaterial-based nanothermometers}

In addition to the above nano-diamonds, there appear a variety of nanomaterials which can be utilized to realize nano-thermometry. One

(a)
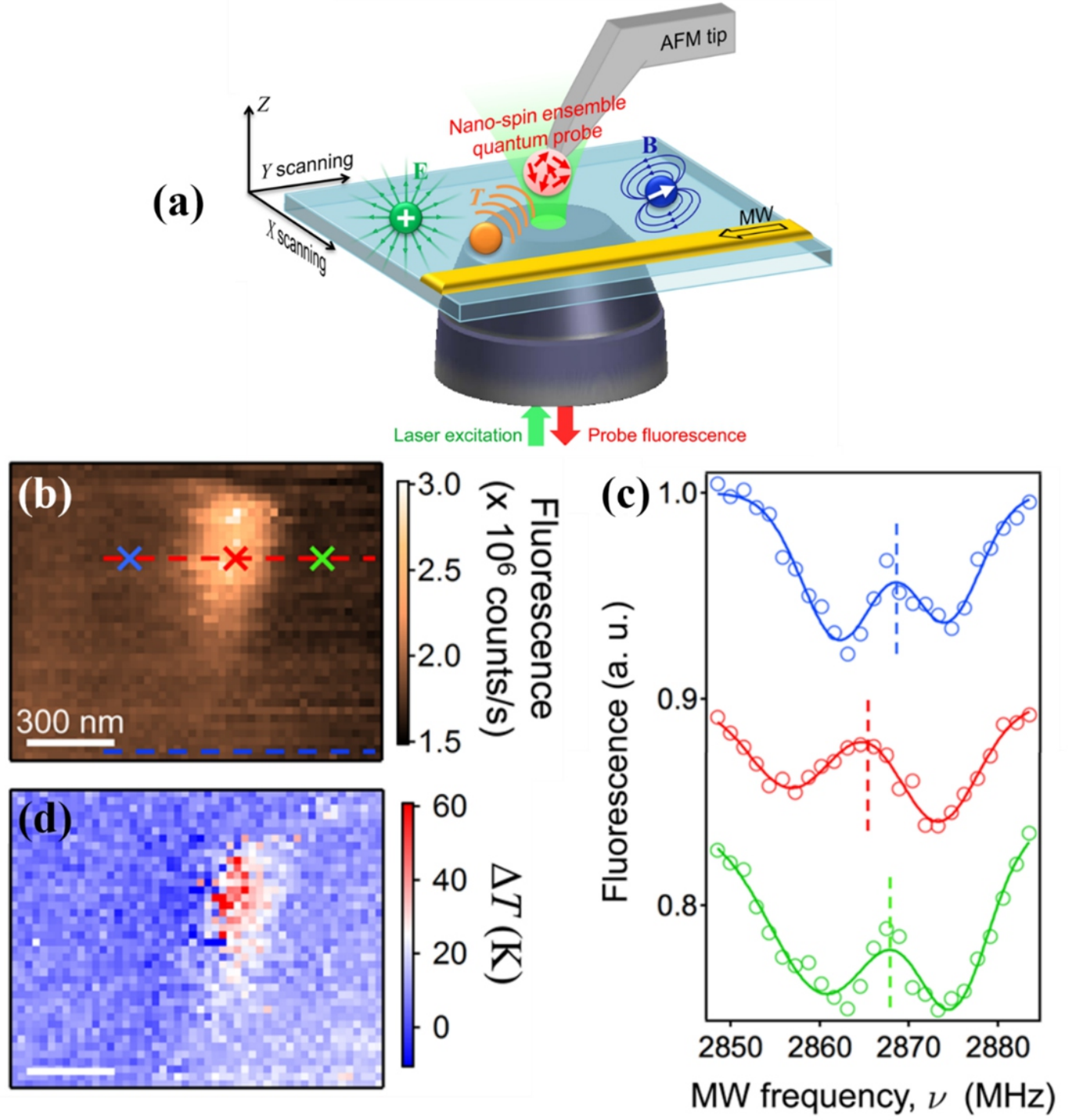

Fig. 7 (a) Schematic of scanning nanodiamond microscopy. (b) Fluorescence image of the $40 \mathrm{~nm}$ gold nanoheater. (c) Spin resonance spectra measured at three different pixels as indicated in (b) with corresponding color. (d) Temperature map deduced from the splitting parameter. ${ }^{76}$ Reprinted with permission from Ref. [77]. Copyright 2016, American Chemical Society. 
example is quite similar to a normal mercurial thermometer and can be suitable for applications in a wide variety of microenvironments. ${ }^{81}$ It consists of a carbon nanotube containing liquid gallium inside (Fig.8(a)), and its height reflects the temperature. To read the temperature, this nanothermometer requires microscopic examination of the carbon nanotube and the gallium level with a scanning electron microscope.

Since the carbon nanotube technique is quite inconvenient and not applicable in wide areas, several other approaches have been attempted to detect temperature in cells via emission intensity or lifetime of organic dyes ${ }^{82,83}$ and transition-metal ions, ${ }^{84,85}$ and they all can provide a result of average temperature for a single cell. Besides, J-M Yang et al. proposed that quantum dot is better because of its brighter exhibition, broader excitation profile for multiplexing and better photostability than organic dyes An instrumental setup is schematically shown in Fig. 8(b) ${ }^{86}$ The temperature sensitivity of quantum dots is basically immune to environment variations. Furthermore, rare-earth nanothermometers are also widely studied, especially in aqueous media or in vivo. Similar to quantum dots, rare-earth nanothermometers also use spectral methods to detect temperature. Note that there are three different biological optical transparency windows (BW; I: 750-950 nm, II: 1000-1350 nm, III: $1500-1800 \mathrm{~nm}$ ) for near-infrared (NIR). ${ }^{87-89}$ BW-I is commonly exploited for effective NIR laser excitation, ${ }^{90,91} \mathrm{BW}-\mathrm{II}$ is better for NIR imaging at greater tissue depths, ${ }^{92,}$ and BW-III offers improved imaging contrast. ${ }^{88,89,9496}$ So it is better to excite nanothermometer in BW-I, while collect the signal in BW-II and BW-III. ${ }^{97}$ A. Skripka et al. ${ }^{98}$ made a multilayered system of $\beta$-phase $\mathrm{NaGdF}_{4}$ nanoparticles that were doped with $\mathrm{Er}^{3+}, \mathrm{Ho}^{3+}, \mathrm{Yb}^{3+}$ and $\mathrm{Nd}^{3+}$ (Fig. 8(d)). $\mathrm{Nd}^{3+}$ were excited by

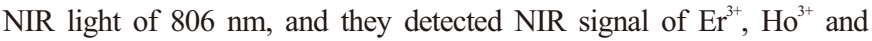
$\mathrm{Nd}^{3+}$. The sensitivity can be around $1.1 \%{ }^{\circ} \mathrm{C}^{-1}$ at the range of $20-50{ }^{\circ} \mathrm{C}$ via using $\mathrm{Ho}^{3+}-\mathrm{Nd}^{3+}$ or $\mathrm{Er}^{3+}-\mathrm{Nd}^{3+} \mathrm{NIR}$ emission band ratios. F-Y Xu et al. ${ }^{99}$ used $\mathrm{NaYF}_{4}: \mathrm{Yb}, \mathrm{Er} @ \mathrm{NaGdF}_{4}: \mathrm{Nd}$ nanoparticles as their nanothermometers, and detected the emission of visible and NIR, getting the result that sensitivity is $0.93 \% \mathrm{~K}^{-1}$ (visible) and $0.46 \% \mathrm{~K}^{-1}$ (NIR) at the range of 283-338 K. In vivo, M. Xu et al. combined triplet-triplet annihilation mechanism with $\mathrm{NaYF}_{4}$ : Nd nanophosphors as their nanothermometer and get high thermal sensitivity $\left(\sim 7.1 \% \mathrm{~K}^{-1}\right)$ and resolution $(\sim 0.1 \mathrm{~K})$ (instrument is shown in Fig. $8(\mathrm{c})) .^{100}$ What's more, DNA can also be applied to make nanothermometer, and it can respond linearly up to $50{ }^{\circ} \mathrm{C}$ in temperature range (Fig. 8(e)). ${ }^{101}$

Although the nanomaterials can work as native and agile marker to measure local temperature, the imaging capability of these nanomaterial-based thermometry appears to be limited because of the uncontrollable distributions of nanomaterials. It is therefore desirable to have controllable local probes in the imaging thermometry. For this sake, scanning probe microscope (SPM) can be very much helpful as we can see in the following two sections (scanning thermal microscope and scanning noise microscope).

\section{Scanning thermal microscope (SThM) 8.1 Principle}

Inheriting from SPM such as scanning tunneling (STM) and atomic force microscopy (AFM), scanning thermal microscopy (SThM) appears as a promising nanoscale thermal imaging technique with excellent spatial resolution $(\sim 10 \mathrm{~nm})$. Williams and Wickramasinghe ${ }^{102}$ first developed the thermocouple integrated scanning probes to obtain the topography of electrically insulating surfaces by using thermal signals as feedback. Although they were not aiming at measuring the thermal distribution, their idea facilitated the development of SThM. So far, AFM-based SThM is widely applied to different materials including metals, semiconductors and insulators. ${ }^{103-105}$ Different from standard AFM, the cantilever probe in SThM is integrated with a thermal sensor and related electronic modules record the temperature distribution of the sample surface as well as the topography when the probe is scanned. The thermal sensors are usually thermocouples, thermistors, or Schottky diodes. $^{106-108}$ SThM typically has two measurement modes, the active
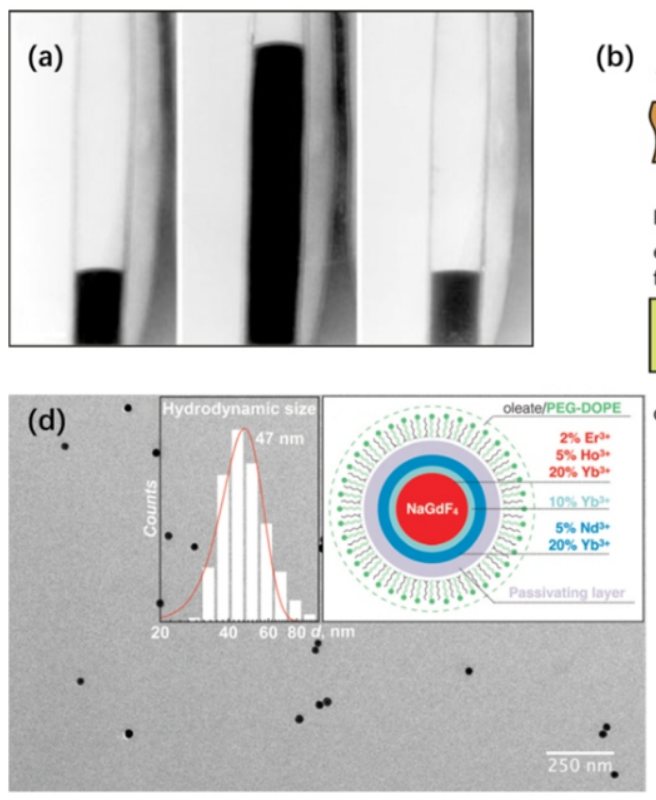

(b)

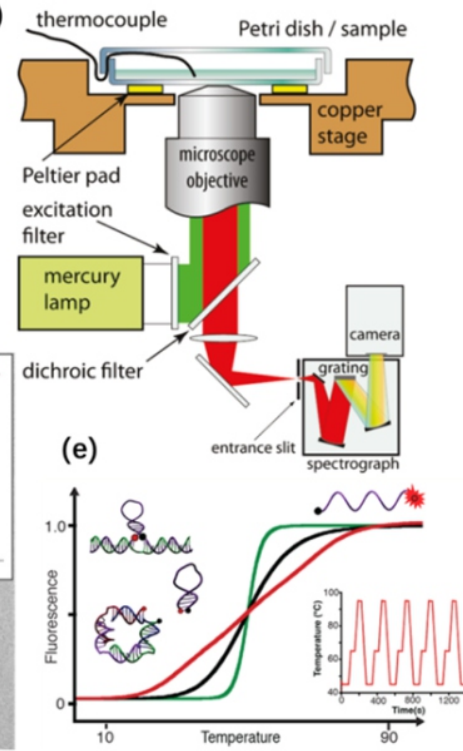

(c)

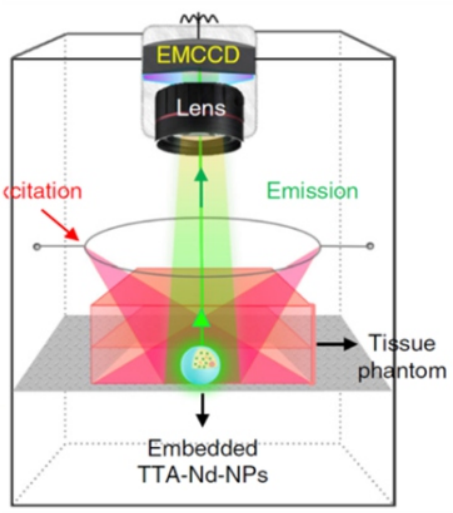

Fig. 8 Several types of nanothermometer: (a) carbon nanotube with gallium; ${ }^{81}$ Reprinted with permission from Ref. [81]. Copyright 2002, Springer Nature. (b) quantum dots nanothermometer; ${ }^{86}$ Reprinted with permission from Ref. [86]. American Chemical Society. (c) nanothermometer based on triplet-triplet annihilation mechanism; ${ }^{100}$ Reprinted with permission from Ref. [100]. Copyright 2018, Springer Nature. (d) nanoparticles doped with Er ${ }^{3+}$, $\mathrm{Ho}^{3+}, \mathrm{Yb}^{3+}$ and $\mathrm{Nd}^{3+}$ and their TEM image; ${ }^{98}$ Reprinted with permission from Ref. [98]. Copyright 2017, The Royal Society of Chemistry. (e) when temperature changes, changes in the molecular structure of the DNA cause changes in fluorescence intensity. ${ }^{101}$ Reprinted with permission from Ref. [101]. Copyright 2016, American Chemical Society. 
mode is to measure the thermal conductivity of the sample, and the passive operation mode is to measure the temperature distribution on the surface of the sample. SThM measurement is often operated in contact mode although tapping mode is also possible. Quantitative measurement with SThM requires a sophisticated calibration process.

\subsection{Applications of scanning thermal microscopy}

Different from the IR emission spectroscopy mentioned above which is limited in far field detection and poor resolution, AFM-based SThM can be used in near-field to directly map the spatial distribution of temperature or thermal conductivity or to explore the fascinating phenomena in the thermal near-field. ${ }^{10,108}$ For example, fluctuational electrodynamics theories ${ }^{109,110}$ reveal that the super-Planck thermal transport dominated by phonon transport will occur in the extreme near field (gap size $<10 \mathrm{~nm}$ ). SThM with a high stiffness probes $\left(\sim 10^{4} \mathrm{Nm}^{-1}\right)$ which can enable measurements down to angstrom-sized gaps can be used to investigate the extreme near-field radiative heat transfer. Cui et $a l .^{108}$ used the method for probing the radiative heat transfer between an Au-coated tip and a heated Au substrate under ultrahigh vacuum conditions. The tip structure is shown schematically in Fig. 9(a) and an SEM image, in Fig. 9(b). The thermoelectric voltage $\left(V_{\text {th }}\right)$ from the Au-
$\mathrm{Cr}$ thermocouple and the tunneling current across the nanogap between the sample and the tip are recorded simultaneously. The results reveal that when the gap size is reduced below about $2.5 \mathrm{~nm}$, the thermal conductance increases with the decreasing gap size and is able to reach up to about $30 \mathrm{nWK}^{-1}$ at the minimum distance within the experimental conditions. The spatial resolution of SThM is determined mainly by the size of the tip. Luo et al. ${ }^{111}$ developed a novel fabrication technique which significantly reduces the size of thermocouple to improve the spatial resolution. The temperature sensitivity is related with the materials of the thermocouple and the surroundings. In order to reduce the influence of the thermal transfer from the environment, Shi et al. ${ }^{112}$ utilized $\mathrm{SiN}_{x}$ and $\mathrm{SiO}_{2}$ to fabricate the cantilever and tip, respectively. Operating in ultrahigh vacuum environment ${ }^{113-115}$ is also an effective way to remove the effects of the thermal transport from surroundings. Besides, some special measurement techniques may also benefit the acquisition of high quality spatial distribution of temperature. For example, Li Shi et al. ${ }^{116}$ utilized SThM with a combined contact mode and lift mode operation to study the low-frequency acoustic phonon temperature in graphene, which was challenging through conventional IR emission spectroscopy tool. ${ }^{117}$ The results reveal that the measured acoustic phonon temperature is similar to the phonon scattering (a)

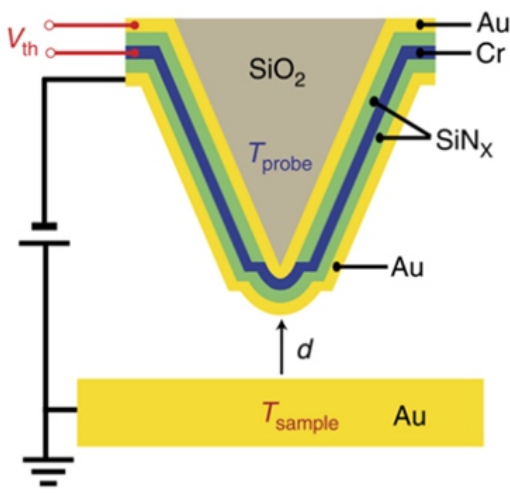

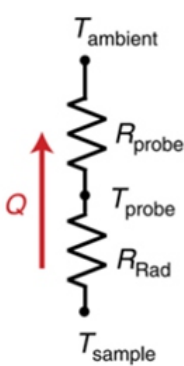

(b)
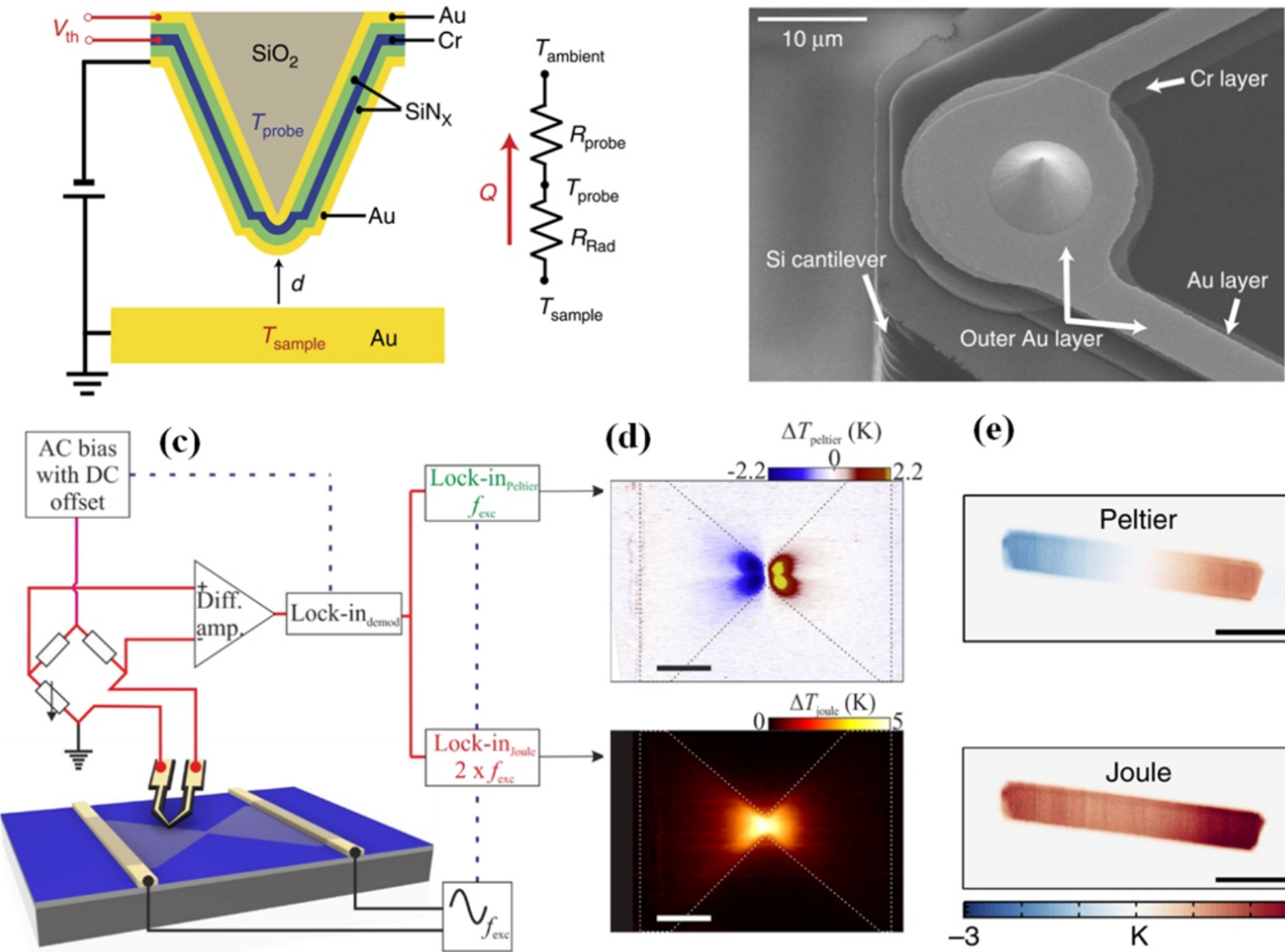

(e)
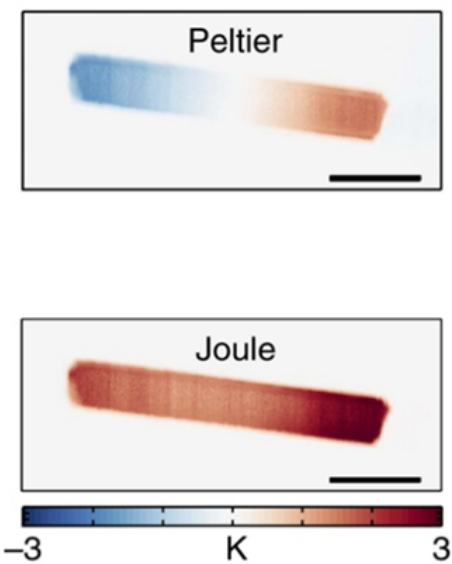

Fig. 9 The schematic (a) and SEM image (b) of a typical Au-Cr thermocouples sensor probe for studying the radiative heat transfer in Angstrom-and nanometer-sized gaps; ${ }^{108}$ Reprinted with permission from Ref. [108]. Copyright 2018, Springer Nature. (c) Schematic of the Scanning Thermal microscopy setup with lock-in technique for studying thermoelectric effect of graphene (as shown in (d) ${ }^{118}$ ) Reprinted with permission from Ref. [118]. Copyright 2018, American Chemical Society. And (e) InAs nanowires. ${ }^{104}$ Reprinted with permission from Ref. [104]. Copyright 2016, Springer Nature. 
temperature determined through the Raman 2D peak shift on the same sample. In 2015, F. Menges et al. ${ }^{104}$ had introduced an AC measurement method to study the thermoelectric effects of a self-heated nanowire (Fig. 9(d)) and later adopted by A. Harzheim et al. ${ }^{118}$ to study the geometrically enhanced thermoelectric effects in graphene nanoconstrictions (Fig. 9(b)). The schematic of AC scheme is shown in Fig. 9(c), using a lock-in amplifier to demodulate and record the thermoelectric signals and hence the temperature distributions. The results demodulated at fundamental harmonic frequency (f) arise from Peltier effect and those demodulated at second frequency (2f), from Joule heating, as demonstrated in the upper (Peltier) and lower (Joule) panels of Figs 9(d:graphene) and (e: InAs nanowire), respectively. In work from Pramod Reddy's group, ${ }^{103}$ they have compared the AC measurement with lock-in with conventional DC scheme for the same samples and significant improvement was found.

In work from our group, we utilized the SThM with an AC scheme to study the near-field electrodynamics in gallium arsenide twodimensional electron gas. GaAs is a direct bandgap semiconductor with two energy valleys and the electrons can be driven to non-equilibrium state by biasing the source and drain. Both experiments and theories ${ }^{119,120}$ have proved that when electrons accumulate enough energy under high electric-fields, as a result they can transfer from (000) valley to (100) valley. By using SThM, we have successfully observed nonlocal hot spots of GaAs lattice which originates from the nonlocal energy dissipation of transporting hot electrons ${ }^{120}$ and thereby heating to the lattice through electron-phonon interaction.

\section{Scanning noise microscope (SNoiM)}

\subsection{Principle of SNoiM}

In downscaled nanoelectronic devices, electrons are driven out of equilibrium with hosting lattice, leading to remarkable hot electron effects. ${ }^{121-123}$ To map the local electron temperature of devices at nanoscale, we develop a kind of nearfield scanning optical microscope (NSOM) called scanning noise microscope(SNoiM) ${ }^{120}$ This method can collect terahertz electromagnetic fluctuations (noises) generated by hot electrons in sample with an ultrahigh sensitive detector, and super resolution is achieved by a very sharp metal tip which can scatter the fluctuating electromagnetic evanescent field on the sample surface. Therefore, SNoiM can be regarded as a near-field version of the microscopic infrared thermometer, as schematically shown in Fig. 10(a). Compared with SThM in contact mode, SNoiM has a promising spectroscopic freedom and works in a non-contact mode or tapping mode. The spectroscopic freedom is crucial to avoid the huge background from lattice environment, namely, the detector collects only a narrow band of electromagnetic waves which is away from the phonon resonances of the hosting lattice, such that the electromagnetic local density of states (EM-LDOS) from lattice is at least one order lower than that from electrons. ${ }^{124}$ As a result, the signal collected by SNoiM is dominated by hot electron contributions. The signal given by the detector should be proportional to the energy density of fluctuating electromagnetic evanescent field of hot electrons, scattered by tip. The energy density can be written as

$$
\mathrm{u}\left(z, \omega, T_{S}\right)=\rho(z, \omega)\left[\hbar \omega /\left\{\exp \left(\hbar \omega / k_{B} T_{e}\right)-1\right]\right.
$$

Here, $\mathrm{z}$ represents the distance between tip and sample surface, $\omega$ represents the angular frequency of detected photons, $T_{e}$ represents the temperature of electrons in the sample, $\rho(z, \omega)$ is the local density of states, $\hbar$ is the Planck constant and $k_{B}$ is the Boltzmann constant. Eventually electron temperature imaging can be realized given the fact that electron contribution $\left(T_{e}\right)$ prevails in the final signal.

\subsection{Applications of SNoiM}

In a nano channel fabricated on GaAs semiconductor device, SNoiM provides for the first time the real space image of hot electron temperature distribution and associated non-local energy dissipation. ${ }^{120}$ As shown in Fig. 10(d) and (e), the electron hot spot reaches as high as $\sim 2000 \mathrm{~K}$ which is much higher than lattice and hence very far away from thermal equilibrium with lattice. So far, SNoiM is the only reported technique to map the electron temperature directly and it can be widely used in exploring the rich dynamics of nonequilibrium phenomena in a broad range of nano-materials and nano-devices.

\section{Summary and outlook}

In summary, temperature mapping with high spatial resolution has

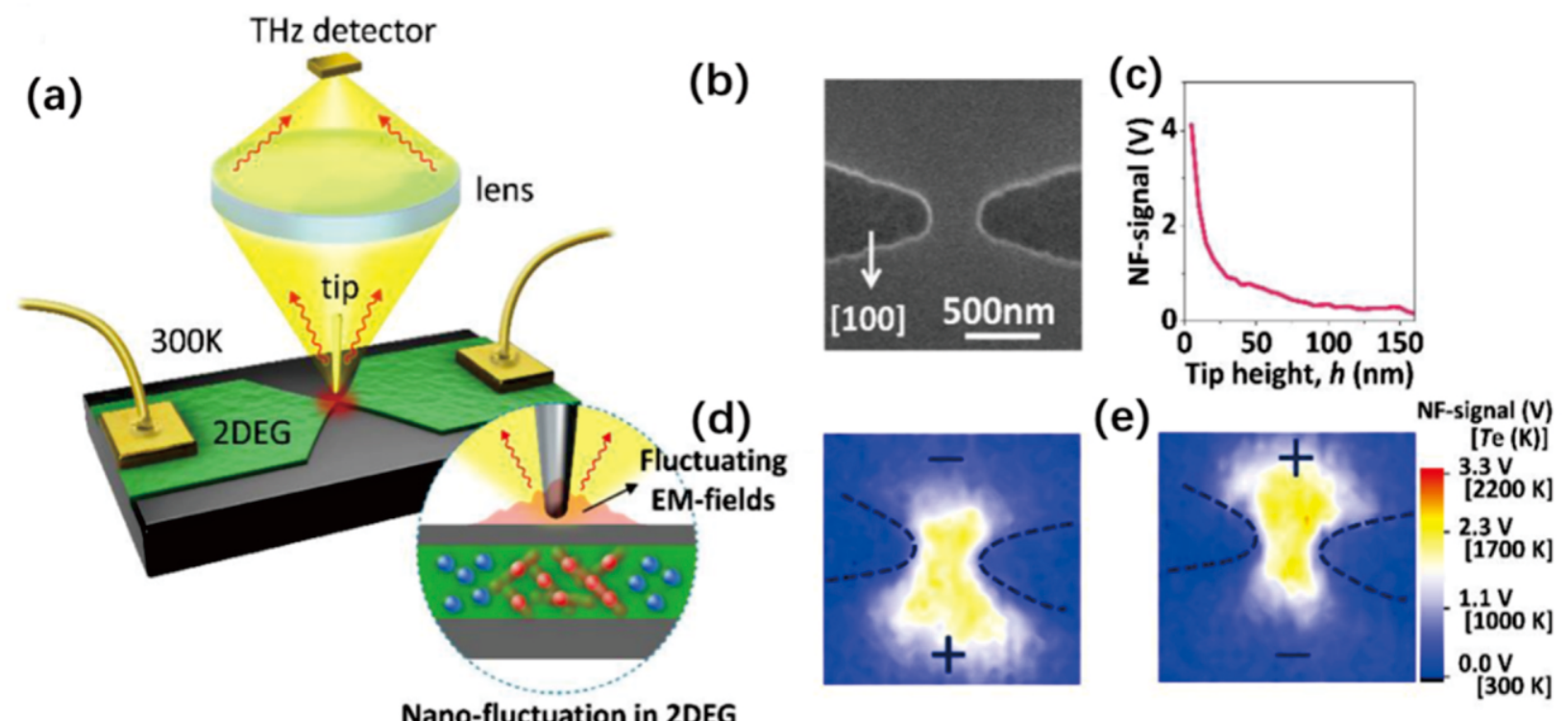

Fig. 10 (a) Schematic of SNoiM. (b) SEM image and (d),(e) SNoiM image of nano-channel fabricated in a GaAs/AlGaAs quantum well (QW) structure. (c) Decay curve of near-field signal with the height of tip. ${ }^{120}$ From [120], Reprinted with permission from AAAS. 


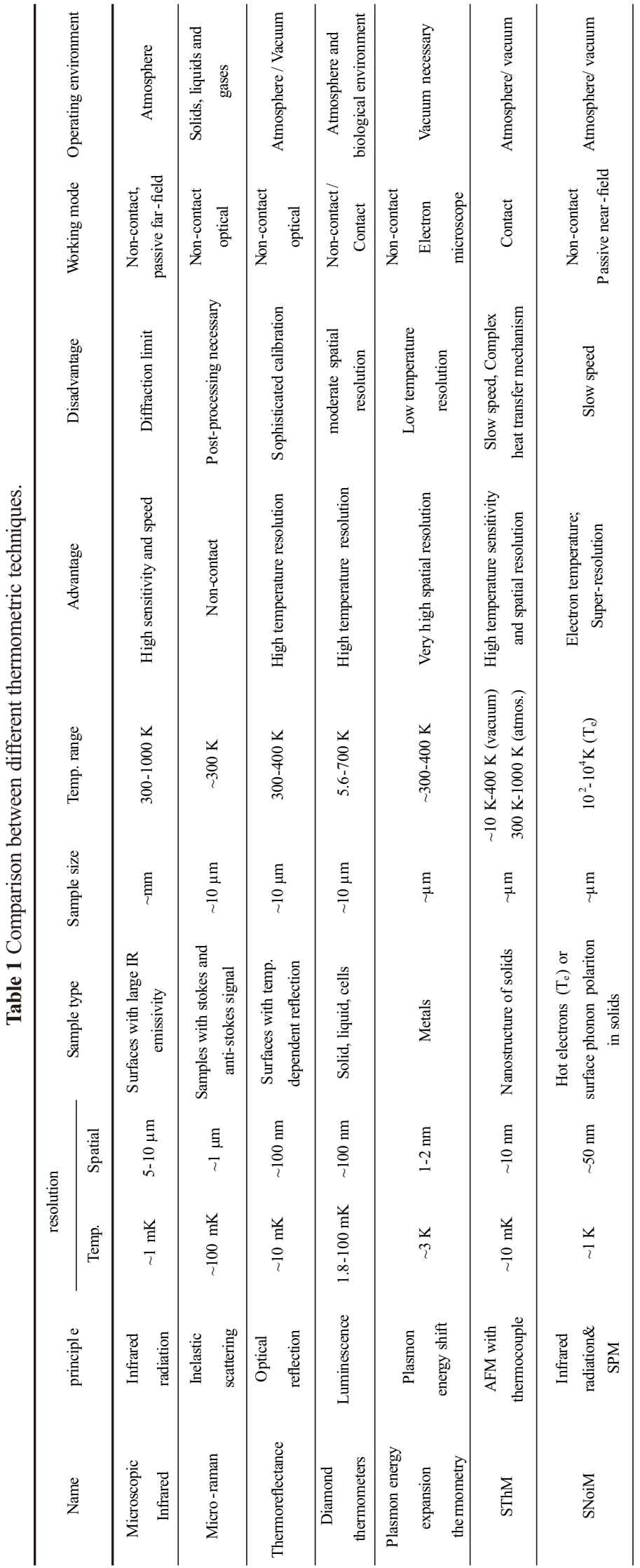

numerous practical applications in micro systems, such as microelectronics, micro-optics, photonics, microfluidics, and nanomedicine. We review various thermometry techniques for micro- and nanosystems and Table 1 summarizes the main features of these techniques. Microscopic infrared thermometer is convenient but restricted in spatial resolution due to diffraction limit; thermoreflectance, micro-Raman use active method with better spatial resolution because of shorter wavelength; plasmon energy expansion thermometry (PEET) achieves very high spatial resolution but requires complicated experiments; Diamond and other nanomaterial-based nanothermometers overcome the diffraction limit and realize high spatial resolution which is determined by size of the nanomaterials; two SPM techniques, namely, scanning thermal microscope (SThM) and scanning noise microscope at terahertz (SNoiM) can also realize the super-resolution imaging capability and SNoiM is so far the only available technique to probe directly electron thermometry. With the rapid development of microand nano-thermometry techniques, much deeper understanding of nanoscale energy or heat transfer and associated non-equilibrium dynamics can be expected and will benefit the development of new nanoelectronic, optoelectronic and thermoelectric devices.

\section{Conflict of interest}

There are no conflicts to declare.

\section{Acknowledgements}

We are grateful to Profs. Wei Lu, S. Komiyama, Drs. Qianchun Weng, Le Yang and Yuexin Zou for fruitful collaborations. Financial supports from the National Key Research Program of China under grant No. 2016YFA0302000, National Natural Science Foundation of China under grant Nos. 11991060/11674070/11427807/ 11634012, and Shanghai Science and Technology Committee under grant Nos. 18JC1420402, 18JC1410300 are also gratefully acknowledged.

\section{References}

1. L. Qin, J. Xu, X. Dong, Q. Pan, Z. Cheng, Q. Xiang and F. Li, Nanotechnology, 2008, 19, 185705.

2. S. Kim, C. B. Roth and J. M. Torkelson, J. Polym. Sci., Part B: Polym. Phys., 2008, 46, 2754-2764.

3. X. Zhao, X. Guo and X. Wang, Fuel Process. Technol., 2007, 88, 237-241.

4. C. Chimerel, L. Movileanu, S. Pezeshki, M. Winterhalter and U. Kleinekathöfer, Eur. Biophys. J., 2008, 38, 121.

5. J. Cao, X. Li, J. Tavakoli and W. X. Zhang, Environ. Sci. Technol., 2008, 42, 3780-3785.

6. A. Narayanaswamy and G. Chen, Appl. Phys. Lett., 2003, 82, 3544-3546.

7. O. Ilic, M. Jablan, J. D. Joannopoulos, I. Celanovic and M. Soljačić, Opt. Express, 2012, 20, A366-A384.

8. D. Polder and M. Van Hove, Phys. Rev. B., 1971, 4, 3303-3314.

9. M. Laroche, R. Carminati and J. J. Greffet, J. Appl. Phys., 2006, 100, 063704.

10. K. Maize, S. R. Das, S. Sadeque, A. M. S. Mohammed, A. Shakouri, D. B. Janes and M. A. Alam, Appl. Phys. Lett., 2015, 106, 5.

11. E. Pop, Nano Res., 2010, 3, 147-169.

12. A. D. Liao, J. Z. Wu, X. Wang, K. Tahy, D. Jena, H. Dai and E. Pop, Phys. Rev. Lett., 2011, 106, 256801.

13. K. L. Grosse, M. H. Bae, F. Lian, E. Pop and W.P. King, Nat. Nanotechnol., 2011, 6, 287-290.

14. J. C. Cuevas, Nat. Commun., 2019, 10, 3342.

15. J. Yang, W. Du, Y. Su, Y. Fu, S. Gong, S. He and Y. Ma, Nat. Commun., 2018, 9, 4033 .

16. A. A. Joshi and A. Majumdar, J. Appl. Phys., 1993, 74, 31-39.

17. H. F.C. Hoevers, M. L. Ridder, A. Germeau, M. P. Bruijn, P. A. J. de Korte and R. J. Wiegerink, Appl. Phys. Lett., 2005, 86, 251903.

18. H. Y. Chiu, V. V. Deshpande, H. W. C. Postma, C. N. Lau, C. Mikó, L. Forró and M. Bockrath, Phys. Rev. Lett., 2005, 95, 226101. 
19. N. Perrin and H. Budd, Phys. Rev. B., 1974, 9, 3454-3463.

20. V. S. Bochkov, T. S. Gredeskul and Y. G. Gurevich, Soviet Physics Journal, 1989, 32, 947-951.

21. X. Xu, J. Zhou and J. Chen, Adv. Funct. Mater, 2019, 1904704.

22. S. Hu, Z. Zhang, Z. Wang, K. Zeng, Y. Cheng, J. Chen and G. Zhang, ES Energy Environ., 2018, 1, 74-79.

23. B. Gao, W. L. Woo and G. Y. Tian, Sci. Rep., 2016, 6, 25480.

24. S. G. Kandlikar, I. Perez-Raya, P. A. Raghupathi, J. L. Gonzalez-Hernandez, D. Dabydeen, L. Medeiros and P. Phatak, Int. J. Heat Mass Transfer, 2017, 108, 2303-2320.

25. S. Daimon, R. Iguchi, T. Hioki, E. Saitoh and K. I. Uchida, Nat. Commun., 2016, 7, 13754

26. O. Wid, J. Bauer, A. Müller, O. Breitenstein, S. S. P. Parkin and G. Schmidt, Sci. Rep., 2016, 6, 28233

27. K. I. Uchida, S. Daimon, R. Iguchi and E. Saitoh, Nature, 2018, 558, 95-99.

28. D. E. Aspnes, P. Handler and D. F. Blossey, Phys. Rev., 1968, 166, 921-933.

29. F. H. Pollak and H. Shen, Mater. Sci. Eng., R, 1993, 10, xv-374.

30. F. H. Pollak, W. Krystek, M. Leibovitch, M. L. Gray and W. S. Hobson, IEEE J. Sel. Top. Quantum Electron., 1995, 1, 1002-1010.

31. H. Shen and M. Dutta, J. Appl. Phys., 1995, 78, 2151-2176.

32. F. H. Pollak, Photonic Probes of Surfaces, Elsevier, Amsterdam, 1995.

33. C. Uher, Semiconductors and Semimetals, Elsevier, 2001

34. O. Acher, S. M. Koch, F. Omnes, M. Defour, M. Razeghi and B. Drévillon, J. Appl. Phys., 1990, 68, 3564-3577.

35. D. U. Kim, K. S. Park, C. B. Jeong, G. H. Kim and K. S. Chang, Opt. Express, 2016, 24, 13906-13916.

36. D. Pierścińska, J. Phys. D: Appl. Phys., 2017, 51, 013001

37. Mansanares A M, Semiconductors and Electronic Materials, Progress in Photothermal and PhotoacousticScience and Technology Series IV, SPIE Optical Engineering, Bellinghamed, 2000.

38. P. W. Epperlein, Temperature, stress, disorder, and crystallization effects in laser diodes: measurements and impacts. SPIE, 1997.

39. D. Pierścińska, K. Pierściński, M. Morawiec, P. Karbownik, P. Gutowski and M. Bugajski, Semicond. Sci. Technol., 2016, 31, 115006.

40. M. Si, C. J. Su, C. Jiang, N. J. Conrad, H. Zhou, K. D. Maize, G. Qiu, C. T. Wu, A. Shakouri, M. A. Alam and P. D. Ye, Nat. Nanotechnol., 2018, 13, 24-28.

41. D. Pierścińska, K. Pierściński, M. Płuska, Ł. Marona, P. Wiśniewski, P. Perlin and M. Bugajski, AIP Adv., 2017, 7, 075107.

42. H. Brugger and P. W. Epperlein, Appl. Phys. Lett., 1990, 56, 1049-1051.

43. P. Y. Yu and M. Cardona, Fundamentals of Semiconductors: Physics and Materials Properties, Springer Berlin Heidelberg, Berlin, Heidelberg, 2010.

44. W. C. Tang, H. J. Rosen, P. Vettiger and D. J. Webb, Appl. Phys. Lett., 1991, 58, 557-559.

45. S. Todoroki, M. Sawai and K. Aiki, J. Appl. Phys., 1985, 58, 1124-1128.

46. P. W. Epperlein, G. L. Bona and P. Roentgen, Appl. Phys. Lett., 1992, 60, 680-682.

47. B. Stoib, S. Filser, J. Stötzel, A. Greppmair, N. Petermann, H. Wiggers, G. Schierning, M. Stutzmann and M. S. Brandt, Semicond. Sci. Technol., 2014, 29, 124005 .

48. M. Mecklenburg, B. Zutter and B. C. Regan, Phys. Rev. Appl., 2018, 9, 7.

49. A. A. Balandin, S. Ghosh, W. Bao, I. Calizo, D. Teweldebrhan, F. Miao and C. N. Lau, Nano Lett., 2008, 8, 902-907.

50. S. Chen, A. Moore, W. Cai, J. Suk, J. An, C. Mishra, C. Amos, C. Magnuson, J. Kang, L. Shi and R. Ruoff, ACS Nano, 2011, 5, 321-328.

51. B. Stoib, S. Filser, N. Petermann, H. Wiggers, M. Stutzmann and M. S. Brandt, Appl. Phys. Lett., 2014, 104, 161907.

52. G. S. Doerk, C. Carraro and R. Maboudian, ACS Nano, 2010, 4, 4908-4914.

53. R. Takahashi and S. Murakami, Semicond. Sci. Technol., 2012, 27, 124005.

54. M. Mecklenburg, W. A. Hubbard, E. R. White, R. Dhall, S. B. Cronin, S. Aloni and B. C. Regan, Science, 2015, 347, 629.

55. G. Meyer, Z. Phys., 1957, 148, 61.

56. H. Abe, M. Terauchi, R. Kuzuo and M. Tanaka, Microscopy, 1992, 41, 465.

57. P. Palanisamy and J. M. Howe, J. Appl. Phys., 2011, 110, 024908.

58. B. C. Regan, W. A. Hubbard, E. R. White, R. Dhall, S. B. Cronin, S. Aloni and M. Mecklenburg, Microsc. Microanal., 2015, 21, 1907.

59. L. M. Pham, D. Le Sage, P. L. Stanwix, T. K. Yeung, D. Glenn, A. Trifonov, P. Cappellaro, P. R. Hemmer, M. D. Lukin, H. Park, A. Yacoby and R. L. Walsworth, New J. Phys., 2011, 13, 045021.
60. F. Dolde, H. Fedder, M. W. Doherty, T. Nöbauer, F. Rempp, G Balasubramanian, T. Wolf, F. Reinhard, L. C. L. Hollenberg, F. Jelezko and J. Wrachtrup, Nat. Phys., 2011, 7, 459.

61. M. P. Ledbetter, K. Jensen, R. Fischer, A. Jarmola and D. Budker, Phys. Rev. A, 2012, 86, 052116.

62. G. Balasubramanian, A. Lazariev, S. R. Arumugam and D. W. Duan, Curr Opin. Chem. Biol., 2014, 20, 69.

63. N. B. Manson, J. P. Harrison and M. J. Sellars, Phys. Rev. B, 2006, 74 104303.

64. F. Jelezko, T. Gaebel, I. Popa, A. Gruber and J. Wrachtrup, Phys. Rev. Lett., 2004, 92, 076401

65. G. Balasubramanian, P. Neumann, D. Twitchen, M. Markham, R. Kolesov, N. Mizuochi, J. Isoya, J. Achard, J. Beck, J. Tissler, V. Jacques, P. R Hemmer, F. Jelezko and J. Wrachtrup, Nat. Mater, 2009, 8, 383.

66. A. Gruber, A. Dräbenstedt, C. Tietz, L. Fleury, J. Wrachtrup and C. V. Borczyskowski, Science, 1997, 276, 2012.

67. R. Schirhagl, K. Chang, M. Loretz and C. L. Degen, Annu. Rev. Phys Chem., 2014, 65, 83.

68. W. W. W. Hsiao, Y. Y. Hui, P. C. Tsai and H. C. Chang, Acc. Chem. Res., 2016, 49, 400 .

69. S. Sotoma, C. P. Epperla and H. C. Chang, ChemNanoMat, 2018, 4, 15.

70. V. M. Acosta, E. Bauch, M. P. Ledbetter, A. Waxman, L. S. Bouchard and D. Budker, Phys. Rev. Lett., 2010, 104, 70801.

71. X. D. Chen, C. H. Dong, F. W. Sun, C. L. Zou, J. M. Cui, Z. F. Han and G. C. Guo, Appl. Phys. Lett., 2011, 99, 161903.

72. D. M. Toyli, D. J. Christle, A. Alkauskas, B. B. Buckley, C. G. Van de Walle and D. D. Awschalom, Phys. Rev. X, 2012, 2, 031001.

73. P. Neumann, I. Jakobi, F. Dolde, C. Burk, R. Reuter, G. Waldherr, J. Honert, T. Wolf, A. Brunner, J. H. Shim, D. Suter, H. Sumiya, J. Isoya and J. Wrachtrup, Nano Lett., 2013, 13, 2738.

74. G. Kucsko, P. C. Maurer, N. Y. Yao, M. Kubo, H. J. Noh, P. K. Lo, H. Park and M. D. Lukin, Nature, 2013, 500, 54.

75. J. Wang, F. Feng, J. Zhang, J. Chen, Z. Zheng, L. Guo, W. Zhang, X. Song, G. Guo, L. Fan, C. Zou, L. Lou, W. Zhu and G. Wang, Phys. Rev. B, 2015, 91, 155404

76. T. Plakhotnik and D. Gruber, Phys. Chem. Chem. Phys., 2010, 12, 9751.

77. J. P. Tetienne, A. Lombard, D. A. Simpson, C. Ritchie, J. Lu, P. Mulvaney and L. C. L. Hollenberg, Nano Lett., 2016, 16, 326.

78. L. Rondin, J. P. Tetienne, P. Spinicelli, C. Dal Savio, K. Karrai, G. Dantelle, A. Thiaville, S. Rohart, J. F. Roch and V. Jacques, Appl. Phys. Lett., 2012, 100, 153118

79. J. Tisler, T. Oeckinghaus, R. J. Stöhr, R. Kolesov, R. Reuter, F. Reinhard and J. Wrachtrup, Nano Lett., 2013, 13, 3152

80. A. W. Schell, P. Engel, J. F. M. Werra, C. Wolff, K. Busch and O. Benson, Nano Lett., 2014, 14, 2623.

81. Y. Gao and Y. Bando, Nature, 2002, 415, 599-599.

82. C. F. Chapman, Y. Liu, G. J. Sonek and B. J. Tromberg, Photochem Photobiol., 1995, 62, 416-425.

83. C. Gota, K. Okabe, T. Funatsu, Y. Harada and S. Uchiyama, J. Am. Chem Soc., 2009, 131, 2766-2767.

84. O. Zohar, M. Ikeda, H. Shinagawa, H. Inoue, H. Nakamura, D. Elbaum, D L. Alkon and T. Yoshida, Biophys. J., 1998, 74, 82-89.

85. M. Suzuki, V. Tseeb, K. Oyama and S. Ishiwata, Biophys. J., 2007, 92, L46L48.

86. J. M. Yang, H. Yang and L. Lin, ACS Nano, 2011, 5, 5067-5071.

87. A. M. Smith, M. C. Mancini and S. Nie, Nat. Nanotechnol., 2009, 4, 710711.

88. E. Hemmer, N. Venkatachalam, H. Hyodo, A. Hattori, Y. Ebina, H Kishimoto and K. Soga, Nanoscale, 2013, 5, 11339-11361.

89. J. Xu, D. Murata, J. Ueda and S. Tanabe, J. Mater. Chem. C, 2016, 4, 11096 11103.

90. A. M. de Grand, S. J. Lomnes, D. S. Lee, M. Pietrzykowski, S. Ohnishi, T. Morgan, A. Gogbashian, R. G. Laurence and J. V. Frangioni, J. Biomed. Opt., 2006, 11, 014007

91. B. delRosal, I. Villa, D. Jaque and F. S. Rodriguez, J. Biophotonics, 2015, 9 , 1059-1067.

92. G. Hong, S. Diao, J. Chang, A. L. Antaris, C. Chen, B. Zhang, S. Zhao, D. N. Atochin, P. L. Huang, K. I. Andreasson, C. J, Kuo and H. Dai, Nat. 
Photonics, 2014, 8, 723-730.

93. G. Hong, J. C. Lee, J. T. Robinson, U. Raaz, L. Xie, N. F. Huang, J. P. Cooke and H. Dai, Nat. Med., 2012, 18, 1841-1846.

94. D. J. Naczynski, M. C. Tan, M. Zevon, B.Wall, J. Kohl, A. Kulesa, S. Chen, C. M. Roth, R. E. Riman and P. V. Moghe, Nat. Commun., 2013, 4, 2199.

95. R. Wang, X. Li, L. Zhou and F. Zhang, Angew. Chem., Int. Ed., 2014, 53, 12086-12090

96. Z. Tao, G. Hong, C. Shinji, C. Chen, S. Diao, A. L. Antaris, B. Zhang, Y. Zou and H. Dai, Angew. Chem., Int. Ed., 2013, 52, 13002-13006

97. E. Hemmer, A. Benayas, F. Legare and F. Vetrone, NanoscaleHoriz., 2016, 1, 168-184.

98. A. Skripka, A. Benayas, R. Marin, P. Canton, E. Hemmer and F. Vetrone, Nanoscale, 2017, 9, 3079-3085.

99. F. Xu, Z. Ba, Y. Zheng, Y. Wang, M. Hu, X. Xu, J. Wang and Z. Zhang, J. Mater Sci., 2018, 53, 15107-15117.

100. M. Xu, X. Zou, Q. Su, W. Yuan, C. Cao, Q. Wang, X. Zhu, W. Feng and F. Yi, Nat. Commun., 2018, 9, 2698.

101. D. Gareau, A. Desrosiers and A. Vallée-Bélisle, Nano Lett., 2016, 16, 39763981.

102. C. C. Williams and H. K. Wickramasinghe, Appl. Phys. Lett, 1986, 49, 1587

103. W. Jeong, K. Kim, Y. Kim, W. Lee and P. Reddy, Sci. Rep., 2014, 4, 4975.

104. F. Menges, P. Mensch, H. Schmid, H. Riel, A. Stemmer and B. Gotsmann, Nat. Commun., 2016, 7, 10874.

105. K. Kim, L. Cui and V. Fernández-Hurtado, Nature, 2015, 528, 387-391.

106. S. Gomès, A. Assy and P. O. Chapuis, Phys. Status Solidi A, 2015, 212, 477.

107. C. Brites, P. Lima, N. Silva, A. Millán, V. Amaral, F. Palacio and L. Carlos, Nanoscale, 2012, 4, 4799-829.

108. L. Cui, W. Jeong, V. Fernandez-Hurtado, J. Feist, F. J. Garcia-Vidal, J. C. Cuevas, E. Meyhofer and P. Reddy, Nat. Commun., 2017, 8, 14479.

109. D. Polder and M. Van Hove, Phys. Rev. B, 1971, 4, 3303-3314.
110. K. Joulain, J. P. Mulet, F. Marquier, R. Carminati and J. J. Greffet, Surf. Sci. Rep., 2005, 57, 59-112.

111. K. Luo, Z. Shi, J. Lai and A. Majumdar, Appl. Phys. Lett., 1996, 68, $325-$ 327.

112. L. Shi, O. Kwon, A. C. Miner and A. Majumdar, J. Microelectromech. Syst., 2001, 10, 370-378.

113. K. Kim, W. Jeong, W. Lee and P. Reddy, ACS Nano, 2012, 6, 4248-57.

114. W. Mueller-Hirsch, A. Kraft, M. Hirsch, J. Parisi and A. Kittel, J. Vac. Sci. Technol., A, 1999, 17, 1205-1210.

115. M. Hinz, O. Marti, B. Gotsmann, M. A. Lantz and U. Dürig, Appl. Phys. Lett., 2008, 92, 043122.

116. I. Jo, I. K. Hsu, Y. J. Lee, M. M. Sadeghi, S. Kim, S. Cronin, E. Tutuc, S. K. Banerjee, Z. Yao and L. Shi, Nano Lett., 2011, 11, 85-90.

117. M. Freitag, H. Y. Chiu, M. Steiner, V. Perebeinos and P. Avouris, Nat nanotechnol., 2010, 5, 497-501.

118. A. Harzheim, J. Spiece, C. Evangeli, E. McCann, V. Falko, Y. Sheng, J. H. Warner, G. A. D. Briggs, J. A. Mol, P. Gehring and O. V. Kolosov, Nano Lett., 2018, 18, 7719-7725.

119. R. D'Agosta, N. Sai and M. Di Ventra, Nano Lett., 2006, 6, 2935-8.

120. Q. Weng, S. Komiyama, L. Yang, Z. An, P. Chen, S. A. Biehs, Y. Kajihara and W. Lu, Science, 2018, 360, 775.

121. K. R. Hofmann, C. Werner, W. Weber and G. Dorda, IEEE Trans. Electron Devices, 1985, 32, 691-699.

122. F. C. Wellstood, C. Urbina and J. Clarke, Phys. Rev. B., 1994, 49, 59425955.

123. R. P. Jindal, IEEE Trans. Electron Devices, 1986, 33, 1395-1397.

124. K. Joulain, R. Carminati, J. P. Mulet and J. J. Greffet, Phys. Rev. B., 2003, 68, 245405.

125. K. Sokolov, Nature, 2013, 500, 36.

Publisher's Note Engineered Science Publisher remains neutral with regard to jurisdictional claims in published maps and institutional affiliations. 